\title{
Anti-parasite drug ivermectin can suppress ovarian cancer by regulating IncRNA-EIF4A3-mRNA axes
}

\author{
$\mathrm{Na} \mathrm{Li} i^{1,2,3} \cdot$ Xianquan Zhan ${ }^{1,2,3,4,5}$
}

Received: 17 February 2020 / Accepted: 7 May 2020 / Published online: 28 May 2020

(C) European Association for Predictive, Preventive and Personalised Medicine (EPMA) 2020

\begin{abstract}
Relevance Ivermectin, as an old anti-parasite drug, can suppress almost completely the growth of various human cancers, including ovarian cancer $(\mathrm{OC})$. However, its anticancer mechanism remained to be further studied at the molecular levels. Ivermectin-related molecule-panel changes will serve a useful tool for its personalized drug therapy and prognostic assessment in OCs.

Purpose To explore the functional significance of ivermectin-mediated lncRNA-EIF4A3-mRNA axes in OCs and ivermectinrelated molecule-panel for its personalized drug therapy monitoring.

Methods Based on our previous study, a total of 16 lncRNA expression patterns were analyzed using qRT-PCR before and after ivermectin-treated different OC cell lines (TOV-21G and A2780). Stable isotope labeling with amino acids in cell culture (SILAC)-based quantitative proteomics was used to analyze the protein expressions of EIF4A3 and EIF4A3-binding mRNAs in ovarian cancer cells treated with and without ivermectin. A total of 411 OC patients from the Cancer Genome Atlas (TCGA) database with the selected lncRNA expressions and the corresponding clinical data were included. Lasso regression was constructed to examine the relationship between lncRNA signature and OC survival risk. The overall survival analysis between highrisk and low-risk groups used the Kaplan-Meier method. Heatmap showed the correlation between risk groups and clinical characteristics. The univariate and multivariate models were established with Cox regression.

Results SILAC-based quantitative proteomics found the protein expression levels of EIF4A3 and 116 EIF4A3-binding mRNAs were inhibited by ivermectin in OC cells. Among the analyzed 16 lncRNAs (HCG15, KIF9-AS1, PDCD4-AS1, ZNF674-AS1, ZNRF3-AS1, SOS1-IT1, LINC00565, SNHG3, PLCH1-AS1, WWTR1-AS1, LINC00517, AL109767.1, STARD13-IT1, LBX2-AS1, LEMD1-AS1, and HOXC-AS3), only 7 lncRNAs (HCG15, KIF9-AS1, PDCD4-AS1, ZNF674-AS1, ZNRF3AS1, SOS1-IT1, and LINC00565) were obtained for further lasso regression when combined with the results of drug testing and overall survival analysis. Lasso regression identified the prognostic model of ivermectin-related three-lncRNA signature (ZNRF3-AS1, SOS1-IT1, and LINC00565). The high-risk and low-risk groups based on the prognostic model were significantly related to overall survival and clinicopathologic characteristics (survival status, lymphatic invasion, cancer status, and clinical stage) in OC patients and remained independent risk factors according to multivariate COX analysis $(p<0.05)$.

Conclusion Those findings provided the potential targeted lncRNA-EIF4A3-mRNA pathways of ivermectin in OC, and constructed the effective prognostic model, which benefits discovery of novel mechanism of ivermectin to suppress ovarian cancer cells, and the ivermectin-related molecule-panel changes benefit for its personalized drug therapy and prognostic assessment towards its predictive, preventive, and personalized medicine (PPPM) in OCs.
\end{abstract}

Electronic supplementary material The online version of this article (https://doi.org/10.1007/s13167-020-00209-y) contains supplementary material, which is available to authorized users.

Xianquan Zhan

yjzhan2011@gmail.com

1 University Creative Research Initiatives Center, Shandong First Medical University, 6699 Qingdao Road, Jinan 250117, Shandong, People's Republic of China

2 Key Laboratory of Cancer Proteomics of Chinese Ministry of Health, Xiangya Hospital, Central South University, 87 Xiangya Road, Changsha 410008, Hunan, People's Republic of China
3 State Local Joint Engineering Laboratory for Anticancer Drugs, Xiangya Hospital, Central South University, 87 Xiangya Road, Changsha 410008, Hunan, People's Republic of China

4 Department of Oncology, Xiangya Hospital, Central South University, 87 Xiangya Road, Changsha 410008, Hunan, People's Republic of China

5 National Clinical Research Center for Geriatric Disorders, Xiangya Hospital, Central South University, 87 Xiangya Road, Changsha 410008, Hunan, People's Republic of China 
Keywords Ovarian cancer $\cdot$ Ivermectin $\cdot \operatorname{lncRNAs} \cdot$ EIF4A3 $\cdot$ TCGA $\cdot$ Prognostic model $\cdot$ Personalized drug therapy $\cdot$ Prognostic assessment $\cdot$ Predictive preventive personalized medicine $($ PPPM)

\section{Introduction}

Avermectin was initially discovered and purified from soil in Japan. Subsequently, avermectin was chemically modified into ivermectin. Compared to avermectin, ivermectin was a much safer and more efficient anti-parasite drug [1]. Ivermectin showed off some amazing promise for global public health in various diseases, which made Drs. Omura and Campbell earn a Nobel Prize in physiology or medicine in 2015 [2]. Ivermectin has demonstrated its antitumor effects in different types of cancers, including ovarian cancer (OC), pancreatic cancer, colon cancer, breast cancer, glioblastoma, melanoma, prostate cancer, lung cancer, and leukemia [3-6]. The antitumor targets of ivermectin include chloride channel, PAK1 protein, NS3 DDX23 helicase, Akt/mTOR pathway, SIN3 domain, multi-drug resistance (MDR) protein, WNTTCF pathway, Nanog/Sox2/Oct4 genes, and P2X7/P2X7 receptors [7-10]. Several antitumor mechanisms of ivermectin have been found. For example, (i) ivermectin can act as an inhibitor of the MDR phenotype by inhibiting the Pglycoprotein pump [11]; (ii) ivermectin causes chloride channels to open and thus trigger cell death [12]; (iii) ivermectin influences the oxidative phosphorylation pathway by inhibiting the mitochondrial complex I [13]; (iv) ivermectin promotes ATP-dependent immune responses through the stimulation of calcium signals [14]; (v) ivermectin promotes cell autophagy and apoptosis through the poly-ubiquitination of the kinase PAK1 [9]; (vi) ivermectin stimulates stem cell pluripotency and preferentially inhibits the cancer stem cell population [15]; and (vii) ivermectin limits the function of tumor progression stimulator DDX23 [16]. Importantly, the in vitro and in vivo antitumor activities of ivermectin can be clinically achievable and can be carried out in clinical trials in healthy or parasitic patients. Safety data of ivermectin treatment with 54 healthy adult volunteers showed some contraindications, such as headache (6.02\% of the study subjects), dysmenorrhea $(5.54 \%)$, throat pain $(1.80 \%)$, and diarrhea $(1.80 \%)$, but no serious adverse events, and ivermectin showed no significant liver and kidney toxicity compared to other drugs [17]. Thus, it is possible for the multi-targeted drug ivermectin from an antiparasitic agent to be a repositioned cancer drug for cancer patients. In terms of clinical and translational science, it is necessary to further study the detailed molecular mechanisms underlying ivermectinmediated suppression of tumor growth, which will clarify the multi-targeted drug mechanism of ivermectin and discover ivermectin-related molecule-panel for its personalized drug therapy in OCs. The ivermectin focusing on Akt/mTOR signaling changes has been reported to benefit for its personalized drug therapy in OCs [18]. The work suggests that ivermectin may be a useful addition to the treatment armamentarium for OC, and that targeting Akt/mTOR signaling is a therapeutic strategy to increase chemosensitivity in OC [18]. Personalized drug therapy is one important aspect of predictive, preventive, and personalized medicine (PPPM) and precision medicine (PM) $[19,20]$. OC is a very complex disease and is involved in a series of molecule alterations that mutually interacted in the molecular network system [21, 22]. Multiomics (including pharmacogenomics)-based molecular network strategy is a promising approach to discover effective multi-therapeutic targets for personalized drug therapy and monitoring [23-25].

Long non-coding RNAs (lncRNAs) with lengths exceeding 200 nucleotides are not translated into proteins, and lncRNAs exert a key role in regulating gene expression such as chromatin remodeling and modification (Xist and Xchromosome inactivation), genomic imprinting (the paternal and maternal chromosome), and gene transcription and posttranscriptional processing (the splicing of mRNA and the generation of endogenous siRNAs) [26, 27]. LncRNAs are up to tens of thousands in mammals according to transcriptomic sequencing by next-generation sequencing, which are extensively involved in many cellular pathogenetic processes, such as tumor suppression, immune response, oncogenesis, apoptosis, autophagy, and cell cycle arrest [28]. OC is the most common and lethal gynecological cancer in women globally. The correlation between lncRNAs and OC may offer novel biomarkers for prognosis, diagnosis, or treatment [29]. Accumulating evidence has recognized the detailed molecular mechanism of lncRNAs in OC, such as their interactions with DNAs, mRNAs, and proteins. LncRNAs can recruit or inhibit transcriptional factors and other transcriptional regulatory proteins to promote or block transcription process of mRNAs according to specific conditions [30]. Eukaryotic translation initiation factor 4A isoform 3 (EIF4A3) is one kind of RNAbinding proteins, serves as a diagnostic marker or therapeutic target for certain types of cancers, and is implicated in many cellular processes, including alteration of ribosome and spliceosome assembly, RNA secondary structure, nuclear and mitochondrial splicing, and translation initiation [31]. Additionally, some drugs have been reported to serve as antitumor effects in epithelial OC cells via lncRNA-EIF4A3mRNA axes [32]. Also, IncRNA-EIF4A3-mRNA axes have been reported in glioma, which may provide a novel strategy for glioblastoma therapy [33]. It is significant to explore ivermectin-mediated lncRNA-EIF4A3-mRNA axes in OC. Our previous isobaric tag for relative and absolute quantification (iTRAQ)-based quantitative proteomics study identified and quantified a total of 5115 proteins from purified mitochondrial samples derived from human OC tissues and 
controls [34]. The key proteins in three energy metabolism pathways (glycolysis, Kreb's cycle, and mitochondrial oxidative phosphorylation pathways) were identified as mitochondrial differentially expressed proteins (mtDEPs), which were the downstream target proteins of IncRNA SNHG3, including PFKM, PKM, PDHB, CS, IDH2, IDH3A, IDH3B, OGDHL, ND2, ND5, CYB, and UQCRH. Further, our study found that IncRNA SNHG3-EIF4A3-energy metabolic key molecules existed and were important for OCs [35]. An expanded transcriptomics data analysis in OC TANRIC database and Starbase database found that 16 lncRNAs (SNHG3, PLCH1AS1, WWTR1-AS1, LINC00517, AL109767.1, ZNRF3AS1, SOS1-IT1, KIF9-AS1, LINC00565, STARD13-IT1, LBX2-AS1, LEMD1-AS1, ZNF674-AS1, PDCD4-AS1, HCG15, and HOXC-AS3) were not only related to OC overall survival but also had binding sites with EIF4A3 [35]. Moreover, stable isotope labeling with amino acids in cell culture (SILAC)-based quantitative proteomics in OC cells treated with and without ivermectin found that the protein expression levels of EIF4A3 and 116 EIF4A3-binding mRNAs were downregulated by ivermectin. It emphasizes the important scientific merits of lncRNAs-EIF4A3-mRNAs in ivermectin-treated OCs.

In this study, the expressions of those 16 lncRNAs were verified with q-PCR, and the protein expression levels of EIF4A3 and EIF4A3-binding mRNAs were verified with SILAC-based quantitative proteomics, in OC cells treated with and without ivermectin. The lasso regression identified the model of three-lncRNA signatures (ZNRF3-AS1, SOS1-IT1, and LINC00565). Those three IncRNAs were related to OC overall survival, and the trend of alerted expression after ivermectin treatment was consistent with patient survival risk. It is the first time to provide lncRNA signature in OC cell lines before and after ivermectin treatment with different drug concentration and provide the correlation of risk score and clinical traits according to prognosis model. Cancer is a complex disease controlled by multiple genes and many signaling pathways, so a key molecule-panel is necessary for personalized drug therapy practice [21]. Constructing ivermectin-mediated multi-lncRNA prognosis model could be reliable and effective for OC prognostic assessment towards PPPM clinical practice. These findings demonstrate a novel link between ivermectin and the IncRNA-EIF4A3-mRNA axes in OCs, indicating that the use of ivermectin may be a new therapeutic approach for OCs.

\section{Materials and methods}

\section{Extraction and preprocessing of TCGA data of OC patients}

The Cancer Genome Atlas (TCGA) data portal provided a platform for researchers to search, download, and analyze datasets of interests (http://cancergenome.nih.gov/). Level 3 RNA-seq V2 data and the corresponding clinical data of OC patients $(n=411)$ were obtained from the TCGA platform. Of them, the clinical data included survival status $(0=$ alive, and $1=$ dead), survival time (days), PANCAN (Pan-Cancer Atlas) , additional radiation therapy (yes/no), age at initial pathologic diagnosis (aged from 30 to 87), anatomic neoplasm subdivision (right, left, and bilateral), clinical stage (stages IIA, IIB, IIC, IIIA, IIIB, IIIC, and IV), lymphatic invasion (yes/no), neoplasm histologic grade (G1, G2, G3, G4, and GX), cancer status (with tumor or tumor-free), primary therapy outcome success (complete remission/response, partial remission/response, progressive disease, and stable disease), and tumor residual disease (no macroscopic disease, 1-10 mm, 11$20 \mathrm{~mm}$, and $>20 \mathrm{~mm}$ ). Survival analysis of lncRNAs in OCs was obtained with TANRIC (http://ibl.mdanderson.org/ tanric/design/basic/index.html). The EIF4A3-binding mRNAs were predicted from the large-scale CLIP-Seq data with starBasev 2.0 (http://starbase.sysu.edu.cn/mirCircRNA. php). Survival analysis of mRNAs in OCs was obtained with the Kaplan-Meier method compared to the log-rank test. The $p$ value $<0.05$ was considered as statistical significance. The binding mRNAs of EIF4A3 that was associated with patient survival rates were further decoded by pathway enrichment with GenCLiP 3 (http://ci.smu.edu.cn/genclip3/analysis. php).

\section{Cell culture and ivermectin treatment}

OC cell lines (TOV-21G and A2780) were purchased from Keibai Academy of Science (Nanjing, China). TOV-21G and A2780 cells were cultured in RPMI-1640 medium (Corning, NY, USA) supplemented with $10 \%$ fetal bovine serum (FBS, GIBCO, South America, NY, USA). All these cells were maintained with $5 \% \mathrm{CO}_{2}$ atmosphere at $37{ }^{\circ} \mathrm{C}$. The cells TOV-21G and A2780 were seeded in 6-well plates and treated with ivermectin $(0 \mu \mathrm{M}, 10 \mu \mathrm{M}, 20 \mu \mathrm{M}, 30 \mu \mathrm{M}, 40 \mu \mathrm{M}$, $50 \mu \mathrm{M}$, and $60 \mu \mathrm{M}$ ) at $70 \%$ density for $24 \mathrm{~h}$.

\section{Effects of ivermectin on OC migration}

The OC cells TOV-21G and A2780 were seeded in 96-well plates $(5000 \mathrm{cell} / \mathrm{well})$ and treated with ivermectin $(0 \mu \mathrm{M}$, $10 \mu \mathrm{M}, 20 \mu \mathrm{M}, 30 \mu \mathrm{M}, 40 \mu \mathrm{M}, 50 \mu \mathrm{M}$, and $60 \mu \mathrm{M})$ for $24 \mathrm{~h}$. CCK8 assay was used to detect the IC50 of ivermectin in A2780 and TOV-21G with different concentration gradients $(0-60 \mu \mathrm{M})$ of ivermectin. Wound healing assay was used to detect the ability of OC migration in each group. The cells TOV-21G and A2780 were seeded in 6-well plates. When cells were grown to approximately $90 \%$ confluency, an artificial wound was created with a $10-\mu l$ pipette tip, and then treated with ivermectin $(0 \mu \mathrm{M}, 10 \mu \mathrm{M}, 20 \mu \mathrm{M}$, and $30 \mu \mathrm{M})$ for $24 \mathrm{~h}$. The cells were then cultured in fresh medium without 
FBS. To visualize wound healing, images were taken at $0 \mathrm{~h}$ and $24 \mathrm{~h}$. The relative percentage of wound healed was calculated as (the width of wound at $0 \mathrm{~h}$ - the width of wound at $24 \mathrm{~h}$ ) / the width of wound at $0 \mathrm{~h}$.

\section{RNA extraction and qRT-PCR verification}

Total RNAs were extracted from each cell line treated with ivermectin $(0 \mu \mathrm{M}, 10 \mu \mathrm{M}, 20 \mu \mathrm{M}$, and $30 \mu \mathrm{M})$ with TRizol ${ }^{\circledR}$ Reagent (Invitrogen, CA, USA) according to the manufacturer's instruction. After the preliminary quantitative analysis of total RNAs, each sample was reversely transcribed into cDNAs for quantitative real-time PCR (qRT-PCR) with SYBR ${ }^{\circledR}$ Premix Ex Taq ${ }^{\mathrm{TM}}$ (TaKaRa, Dalian, China). The qRT-PCR was used to measure the expressions of lncRNAs (HCG15, KIF9-AS1, PDCD4-AS1, ZNF674-AS1, ZNRF3AS1, SOS1-IT1, LINC00565, SNHG3, PLCH1-AS1, WWTR1-AS1, LINC00517, AL109767.1, STARD13-IT1, LBX2-AS1, LEMD1-AS1, and HOXC-AS3) in cells TOV$21 \mathrm{G}$ and $\mathrm{A} 2780$ before and after treatment with ivermectin $(0 \mu \mathrm{M}, 10 \mu \mathrm{M}, 20 \mu \mathrm{M}$, and $30 \mu \mathrm{M})$. Beta-actin was used as an internal control for mRNA quantification. The primer sequences of 16 lncRNAs for qRT-PCR analysis were summarized (Table 1).

\section{SILAC labeling}

SILAC labeling used kits from Thermo Fisher Scientific with RPMI 1640 lacking lysine and arginine supplemented with $100 \mathrm{mg} / 1\left[{ }^{13} \mathrm{C}_{6},{ }^{15} \mathrm{~N}_{4}\right]$ arginine and $100 \mathrm{mg} / 1\left[{ }^{13} \mathrm{C}_{6},{ }^{15} \mathrm{~N}_{2}\right]$ lysine with $10 \%$ dialyzed fetal bovine serum. TOV-21G OC cells were cultured with normal RPMI 1640 and heavy chain labeled RPMI 1640. After 8 passages, TOV-21G cells cultured with heavy chain labeled RPMI 1640 were treated with $20 \mu \mathrm{M}$ ivermectin. TOV-21G cells were cultured with normal RPMI 1640 treated with DMSO. Cells were collected after $24 \mathrm{~h}$ treatment for protein extraction. The extracted proteins from TOV-21G cell treated with and without ivermectin were digested with trypsin, followed by peptide fractionation ( $n=15$ fractions), liquid chromatography (LC)-tandem mass spectrometry (MS/ MS), and database searching to identify and quantify proteins in OC cells treated with and without ivermectin.

\section{Lasso regression for $\mathrm{OC}$ tissues}

Least absolute shrinkage and selection operator (lasso) regression was established to examine the relationship of IncRNA signature and $\mathrm{OC}$ survival risk with the glmnet R package. Lasso regression has been proved to have good curve fitting characteristics and relatively high precision models. A more refined model can be obtained after compressing some regression coefficients by adjusting parameter lambda. Best subset selection and the connections between lasso coefficient
Table 1 The primer sequence of 16 lncRNAs for qRT-PCR assessment in $\mathrm{OC}$ cell lines. $\mathrm{F}=$ forward; $\mathrm{R}=$ reverse

\begin{tabular}{|c|c|}
\hline Primer name of lncRNAs & Primer sequence (from $5^{\prime}$ to $3^{\prime}$ ) \\
\hline SNHG3-F & СTCTACTACCTGGCGCACCT \\
\hline SNHG3-R & CССССТTCAGTCCCATCCTT \\
\hline HCG15-F & GTGTTAGCCAGGATGGTCTCGATC \\
\hline HCG15-R & CGGTAGCTCACGCCTGTAATCTC \\
\hline KIF9-AS1-F & ACGGCTGCTGAGGTCTGAGTC \\
\hline KIF9-AS1-R & AAGGCACCAAGCACTGTCACTTC \\
\hline LBX2-AS1-F & GCTGCTGACAGACAAGACCAAGG \\
\hline LBX2-AS1-R & TAATGTGCCGAGGCCGGAGAC \\
\hline PDCD4-AS1-F & AGCTCAACGATCCTCTCACCTCAG \\
\hline PDCD4-AS1-R & TGGCTCACGCTTGTAATCTCAACG \\
\hline WWTR1-AS1-F & CTGTCACTGGAGATGCGGCTTC \\
\hline WWTR1-AS1-R & GTGGTGGCAGGCGTCTTAATCC \\
\hline ZNF674-AS1-F & AGGCTGGTCTCGAACTCCTGAC \\
\hline ZNF674-AS1-R & GCAGTGGCTCACACCTGTAACC \\
\hline ZNRF3-AS1-F & TGAGGTCAGGAGTTCGAGATCAGC \\
\hline ZNRF3-AS1-R & GGTGCCATCTTCGCTCACTACAAC \\
\hline SOS1-IT1-F & GGTTTGTCATCCCAGTCTGCT \\
\hline SOS1-IT1-R & TCGAATTTGACCCCCAGAAGG \\
\hline HOXC-AS3-F & CGCAGAGTGGAGTAACAGCG \\
\hline HOXC-AS3-R & GCGCTCTGTAAAGGACGCTT \\
\hline AL109767.1-F & AGGCTGGTGTCAGGAGGATGTC \\
\hline AL109767.1-R & TGGACTCCTCTGACTGTCTGCTG \\
\hline LEMD1-AS1-F & GCCACTGGTAACTTGCCGTCTAC \\
\hline LEMD1-AS1-R & TTCTGTCCTGGTCTCTGTCAGTCC \\
\hline LINC00517-F & AGTGTTGCATCCTTGCCCTG \\
\hline LINC00517-R & GGGTGAACAGGCTGGAATGG \\
\hline LINC00565-F & CGCAGAGGATCGGCAGCATTC \\
\hline LINC00565-R & TCGCTCTGTGGACCTTGGATGG \\
\hline PLCH1-AS1-F & CTGTTGCTCTGCCAGCCAAGG \\
\hline PLCH1-AS1-R & GAAGCATGGAGGTTCAAGGTCAGG \\
\hline STARD13-IT1-F & ATGGGCTTGCTTACATGGGC \\
\hline STARD13-IT1-R & CCATAGGAGTGGCTGAGGGT \\
\hline
\end{tabular}

estimates can be identified to construct the prognostic model. Further, clinical characteristics (PANCAN, additional radiation therapy, age at initial pathologic diagnosis, anatomic neoplasm subdivision, clinical stage, lymphatic invasion, neoplasm histologic grade, cancer status, primary therapy outcome success, and tumor residual disease) that were associated with overall survival in OC patients were used for Cox regression (univariate model and multivariate model) and Kaplan-Meier method to evaluate the availability of prognostic model.

\section{Statistical analysis}

The student's $t$ test in SPSS 13.0 (SPSS Inc., Chicago, USA) was used to assess the expression differences of lncRNAs in 
cells (TOV-21G and A2780) before and after treatment with ivermectin $(0 \mu \mathrm{M}, 10 \mu \mathrm{M}, 20 \mu \mathrm{M}$, and $30 \mu \mathrm{M})$. Each experiment was repeated at least three times. The relationship of clinical characteristics between different risk sore groups (high-risk group vs. low-risk group) was analyzed with chisquare test. In all cases, $p<0.05$ was considered as statistical significance $(* p$ value $<0.05 ; * * p$ value $<0.01$; and $* * * p$ value $<0.001)$. Benjamini-Hochberg for multiple testing and false discovery rate (FDR) (q-value) were calculated to correct the $p$ value for pathway enrichment analysis and SILACbased quantitative proteomics analysis.

\section{Results}

\section{The overall survival analysis and enriched pathways of EIF4A3-binding mRNAs}

Our previous study identified 1198 mtDEPs in OC tissues relative to controls with iTRAQ-based quantitative proteomics [25]. Among those 1198 mtDEPs, six RNA-binding proteins (IGF2BP2, EIF4A3, C22ORF28, SFRS1, UPF1, and EWSR1) were predicted to have binding sites with IncRNAs. Of them, EIF4A3 was a key molecule, which was identified in energy metabolic pathway-related protein-protein network in OC. Interestingly, 16 lncRNAs (HCG15, KIF9AS1, PDCD4-AS1, ZNF674-AS1, ZNRF3-AS1, SOS1-IT1, LINC00565, SNHG3, PLCH1-AS1, WWTR1-AS1, LINC00517, AL109767.1, STARD13-IT1, LBX2-AS1, LEMD1-AS1, and HOXC-AS3), which were selected with conjoint data analysis of the TANRIC database and Starbase database, were not only related to OC overall survival but also had binding sites with EIF4A3 [35].

This study further analyzed the function, potential mechanism, and overall survival rate of mRNAs binding to EIF4A3. EIF4A3-binding mRNAs were predicted with Starbase, which obtained 3636 EIF4A3-binding mRNAs in various cancers (cancer number >10) (Supplementary Table 1). Furthermore, those mRNAs were used for overall survival analysis, which found that 306 EIF4A3-binding mRNAs were significantly related to survival rate in OCs (Supplementary Table 2). The GenCLiP 3 database analysis of these 306 EIF4A3-binding mRNAs found that 73 EIF4A3-binding mRNAs were previously studied in OCs, including MTOR, NOTCH1, PLAUR, NME1, CDK4, FGF2, HMGB1, MRE11, LEPR, ELAVL1, CFL1, CLIC1, ST3GAL1, JAK1, CALR, MCM3, LRP1, C1QBP, NOTCH2, SRPK1, NUAK1, FAM129B, IGFBP4, CDK14, P4HB, RELB, PBK, MEF2A, CHKA, N4BP1, EXO1, SIRT7, ARHGDIA, CYP27B1, STOML2, TPM3, FANCG, ILF2, STARD13, LIN9, MUL1, MANF, SMUG1, ARHGEF2, PDIA6, DOCK4, PDE4A, PLXNB2, MKL1, TRIP13 BCL7A, HIF1AN, PDIA4, CCDC69, DPP3, BAIAP2L1, ASNA1,
CPEB3, SIAH2, FDPS, LSM2, TXNDC17, RAPGEF3, PRPF4, DFFA, ETFA, UBL5, RPL22L1, CACNA1G, FOXN3, ZBTB7A, PUF60, and PSMB4, and 233 EIF4A3binding mRNAs were not previously studied. Moreover, among those 306 EIF4A3-binding mRNAs, the protein expression levels of 116 EIF4A3-binding mRNAs and EIF4A3 were identified by SILAC-based quantitative proteomics in $\mathrm{OC}$ cells treated with and without ivermectin, and the protein expressions of all these EIF4A3-binding mRNAs and EIF4A3 were inhibited by ivermectin (Table 2). These results demonstrate that our findings were consistent with previous studies and also made new discoveries.

The signaling pathways enriched with 306 EIF4A3binding mRNAs were clustered into 8 functional groups (Fig. 1a and Supplementary Table 3). Cluster 1 functioned in cell cycle. Cluster 2 functioned in purine metabolism and pyrimidine metabolism. Cluster 3 functioned in mitochondrial translation elongation, mitochondrial translation, mitochondrial translation termination, mitochondrial translation initiation, translation, and selenoamino acid metabolism. Cluster 4 functioned in mRNA splicing, processing of capped intron, spliceosome, and metabolism of RNA. Cluster 5 functioned in nucleotide excision repair and transcription-coupled nucleotide excision repair. Cluster 6 functioned in Hedgehog, Notch, retinoblastoma gene in cancer, and metabolism of proteins. Cluster 7 functioned in cargo recognition for clathrinmediated endocytosis and clathrin-mediated endocytosis RNA degradation. Cluster 8 functioned in purine nucleotides nucleosides metabolism, purine metabolism, EGFR1, and metabolism of vitamins and cofactors.

\section{Ivermectin inhibited migration of $\mathrm{OC}$ cells in vitro}

The anticancer ability of ivermectin was measured with CCK 8 assay after ivermectin $(0-60 \mu \mathrm{M})$ treatment for $24 \mathrm{~h}$ in $\mathrm{OC}$ cells A2780 and TOV-21G. IC50 was $20.79 \mu \mathrm{M}$ in A2780 cells and $22.54 \mu \mathrm{M}$ in TOV-21G cells (Fig. 1b). Moreover, wound healing assay found that ivermectin significantly suppressed cell migration of cells A2780 and TOV-21G in a concentration of $20 \mu \mathrm{M}$ and $30 \mu \mathrm{M}$ ivermectin (Fig. $1 \mathrm{c}$ and d).

\section{The screening of IncRNAs being regulated by ivermectin}

It is significant to explore ivermectin-mediated lncRNAEIF4A3-pathway axis in OCs. The qRT-PCR was used to measure the lncRNA expressions (HCG15, KIF9-AS1, PDCD4-AS1, ZNF674-AS1, ZNRF3-AS1, SOS1-IT1, LINC00565, SNHG3, PLCH1-AS1, WWTR1-AS1, LINC00517, AL109767.1, STARD13-IT1, LBX2-AS1, LEMD1-AS1, and HOXC-AS3) in cells A2780 and TOV$21 \mathrm{G}$ after treatment with ivermectin $(0 \mu \mathrm{M}, 10 \mu \mathrm{M}, 20 \mu \mathrm{M}$, 


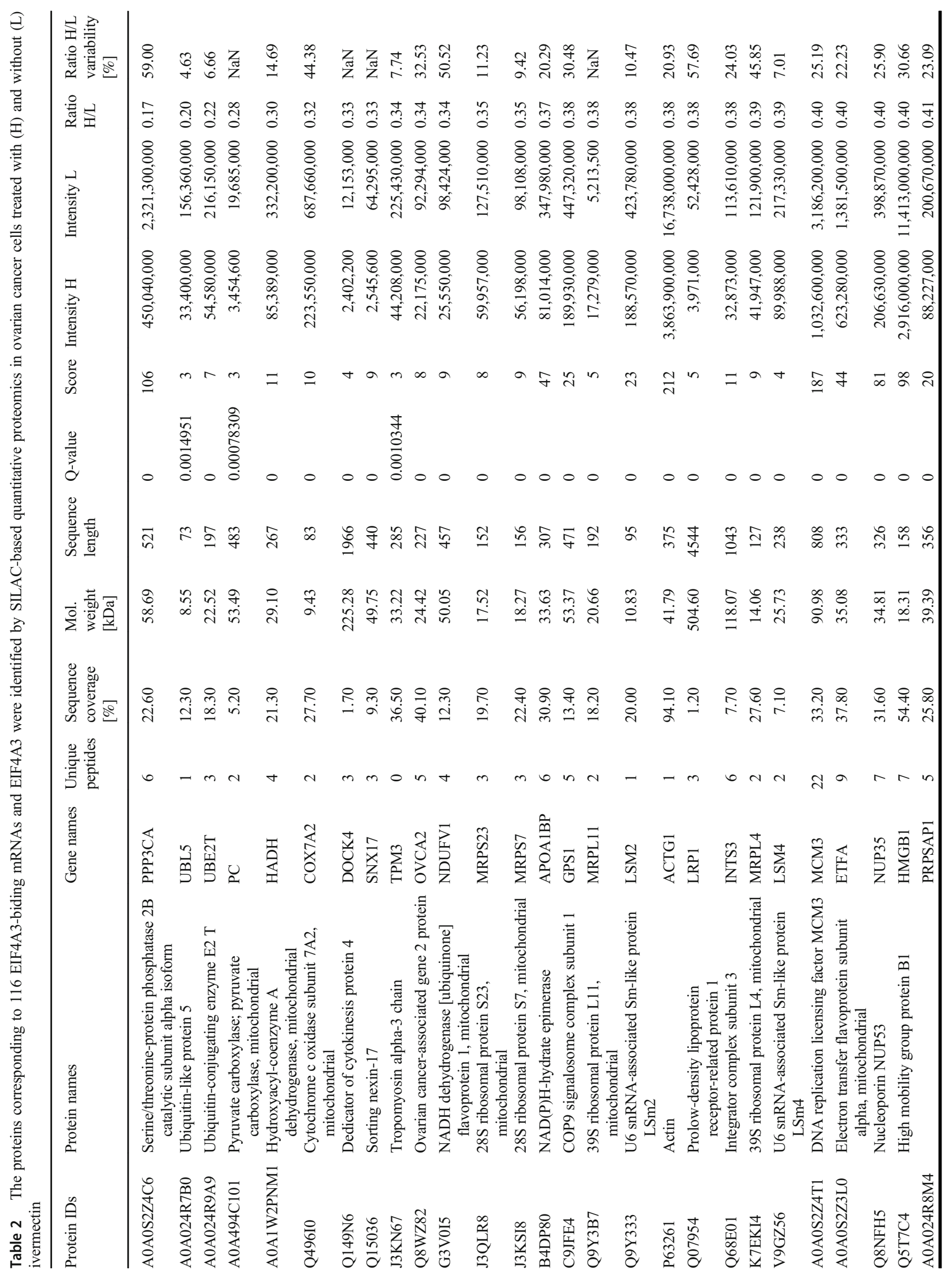




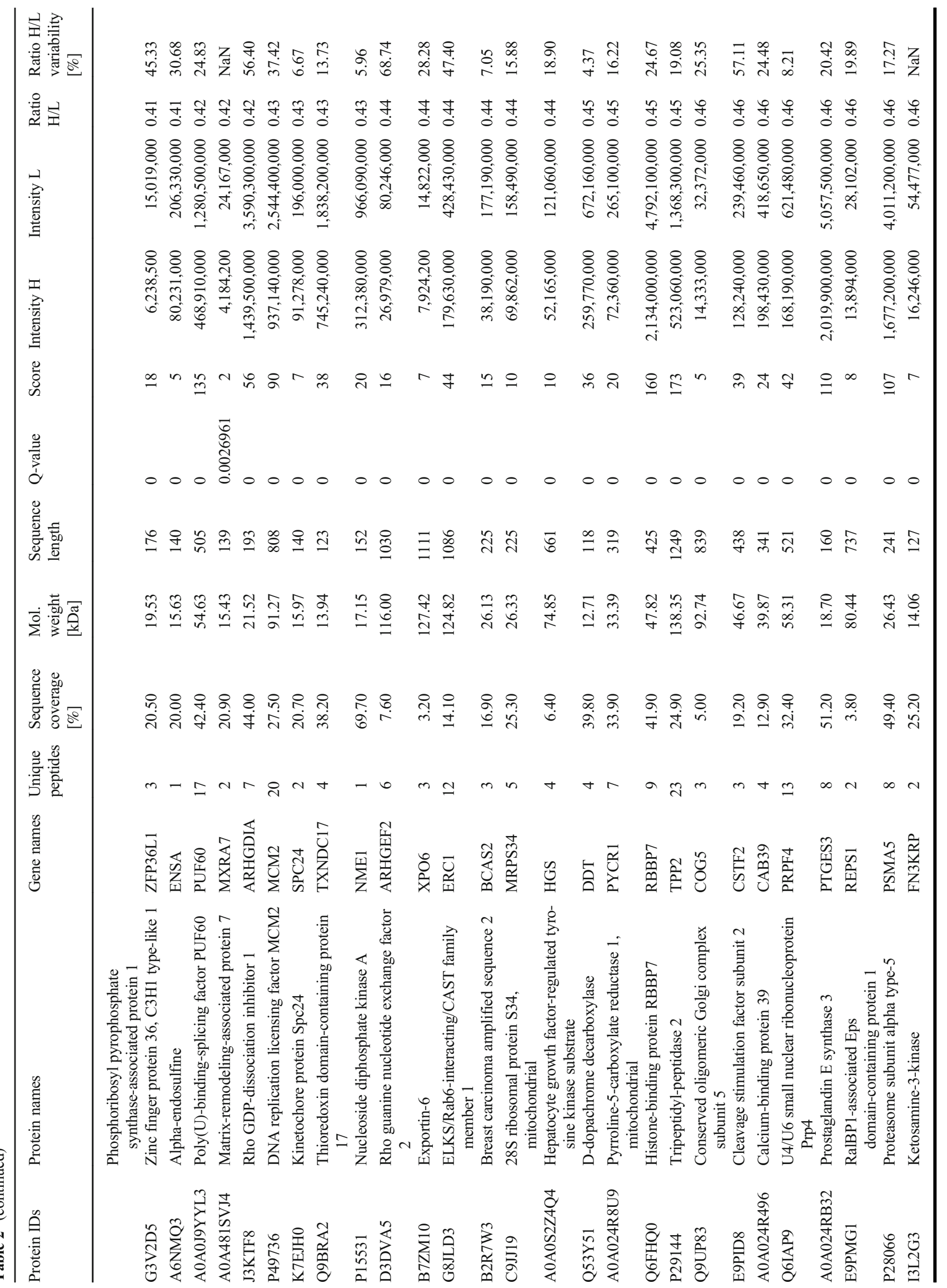




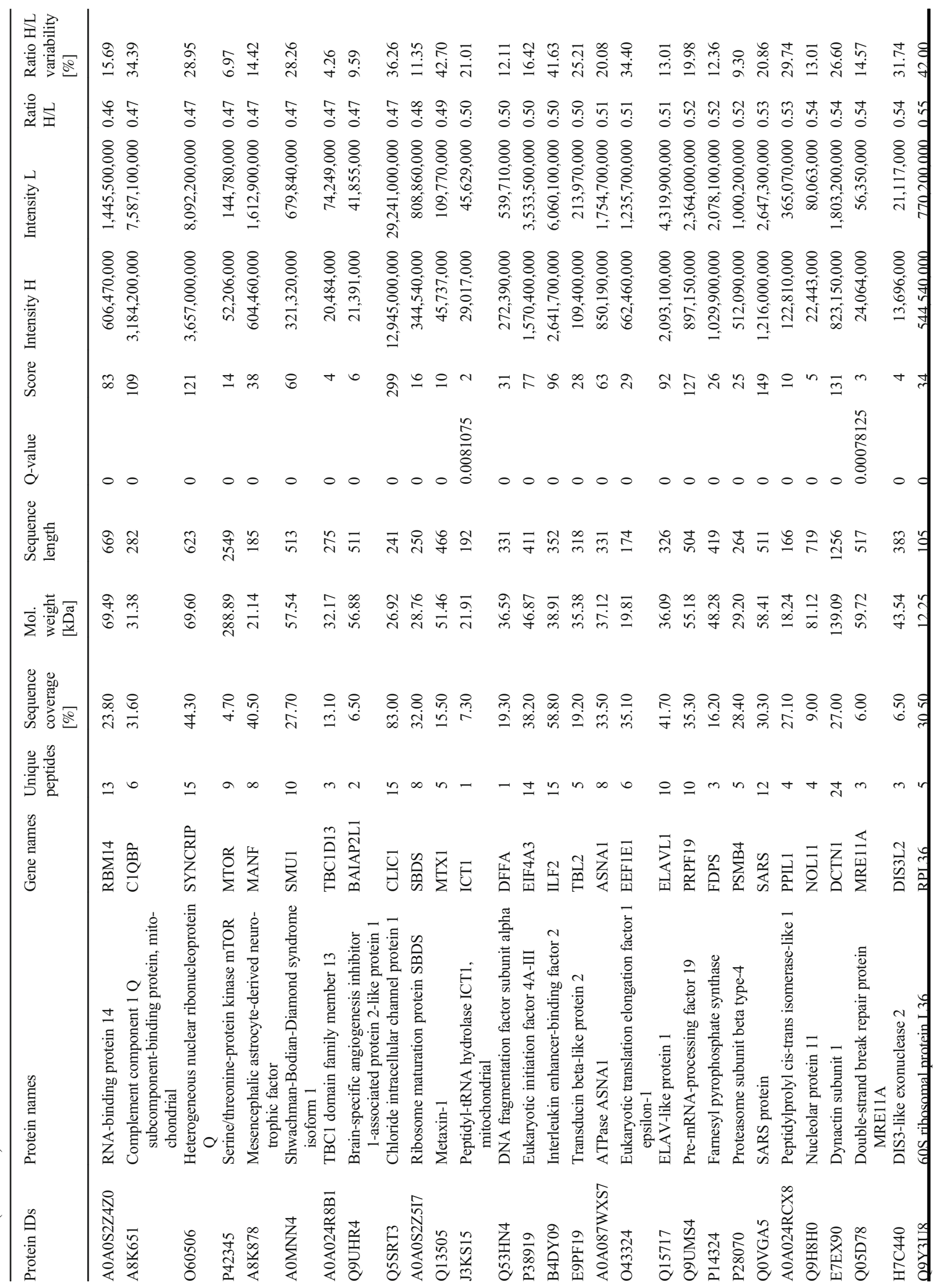




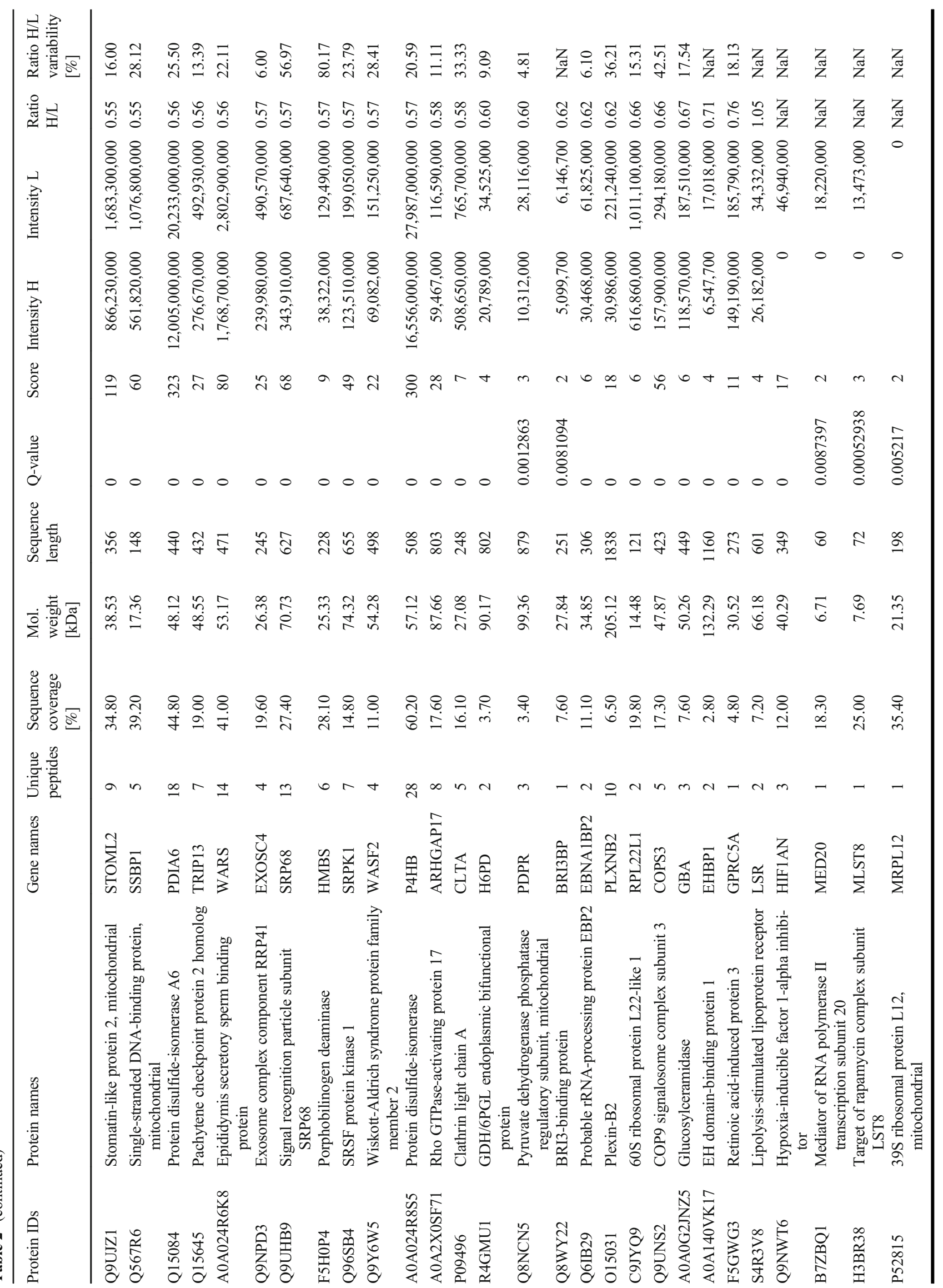




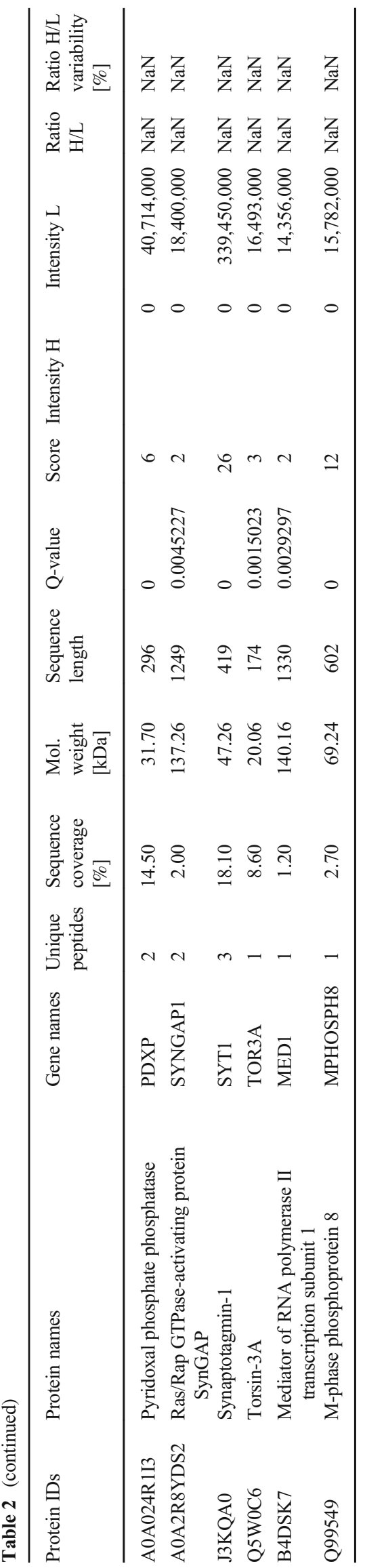

and $30 \mu \mathrm{M})$ (Figs. 2 and 3). The significant changes were found for lncRNA expressions in cells A2780 and TOV$21 \mathrm{G}$, and the changed trend was consistent with survival risk, including HCG15, KIF9-AS1, PDCD4-AS1, ZNF674-AS1, ZNRF3-AS1, SOS1-IT1, LINC00565, AL109767.1, and LBX2-AS1. The expressions of AL109767.1 and LBX2AS1 in cells TOV-21G were too low to be tested by qRTPCR, so those two IncRNAs were excluded for further analysis in TOV-21G.

\section{Lasso regression identified the prognostic model with three-IncRNA signature for OCs}

Totally, seven lncRNAs, including HCG15, KIF9-AS1, PDCD4-AS1, ZNF674-AS1, ZNRF3-AS1, SOS1-IT1, and LINC00565 (Fig. 4), were selected to perform lasso regression (Supplementary Table 4). The alerted trend of overall survival curve of those seven IncRNAs was consistent with the results of ivermectin treatment. Further, the condensed prognostic model of four-lncRNA signature (PDCD4-AS1, ZNRF3AS1, SOS1-IT1, and LINC00565) was identified when log (lambda) was between -3 and -4 (Fig. 5 a and b). Finally, the optimized prognostic model of three-lncRNA signature (ZNRF3-AS1, SOS1-IT1, and LINC00565) was identified according to curve fit coefficient of each lncRNA. Considering the coefficients of PDCD4-AS1 (coefficient = 0.026), ZNRF3-AS1 (coefficient $=-1.720$ ), SOS1-IT1 (coefficient $=-0.206)$, and LINC00565 (coefficient $=0.360)$, PDCD4-AS1 was not suitable to be selected in the optimized prognostic model. The optimized prognostic model of threelncRNA signature showed a statistically significant difference in overall survival between high-risk and low-risk groups (Fig. 5c). Three-lncRNA-signature-based high-risk group was significantly related to the clinical characteristics, including survival status $(0=$ alive and $1=$ dead $)$, clinical stage (stages IIA, IIB, IIC, IIIA, IIIB, IIIC, and IV), lymphatic invasion (yes/no), and cancer status (with tumor or tumor-free) $(p<0.05)$ (Fig. 6; Supplementary Tables 5 and 6).

Furthermore, the risk score of each OC sample based on lasso regression of three-lncRNA signature (Supplemental Table 6) acted as a risk factor to perform univariate and multivariate analyses. The univariate analysis revealed that PANCAN (hazard ratio [HR] 0.91; 95\% confidence interval [CI] 0.84-0.99; $p=2.90 \mathrm{E}-02)$, age at initial pathologic diagnosis (HR 1.02; 95\% CI 1.01-1.03; $p=2.00 \mathrm{E}-04$ ), anatomic neoplasm subdivision (HR 1.34; 95\% CI 1.04-1.74; $p=2.27 \mathrm{E}$ -02 ), cancer status (HR 10.96; 95\% CI 5.13-23.42; $p=$ $6.48 \mathrm{E}-10$ ), primary therapy outcome success (HR 1.55; $95 \%$ CI $1.35-1.78 ; p=2.86 \mathrm{E}-10$ ), tumor residual disease (HR $1.29 ; 95 \%$ CI $1.12-1.49 ; p=3.00 \mathrm{E}-04)$, and risk score (HR 3.09 ; $95 \%$ CI $1.85-5.17 ; p=1.76 \mathrm{E}-05$ ) were significantly correlated with a poor OS (Fig. 7a). The multivariate analysis revealed that age at initial pathologic diagnosis (HR 1.02; 


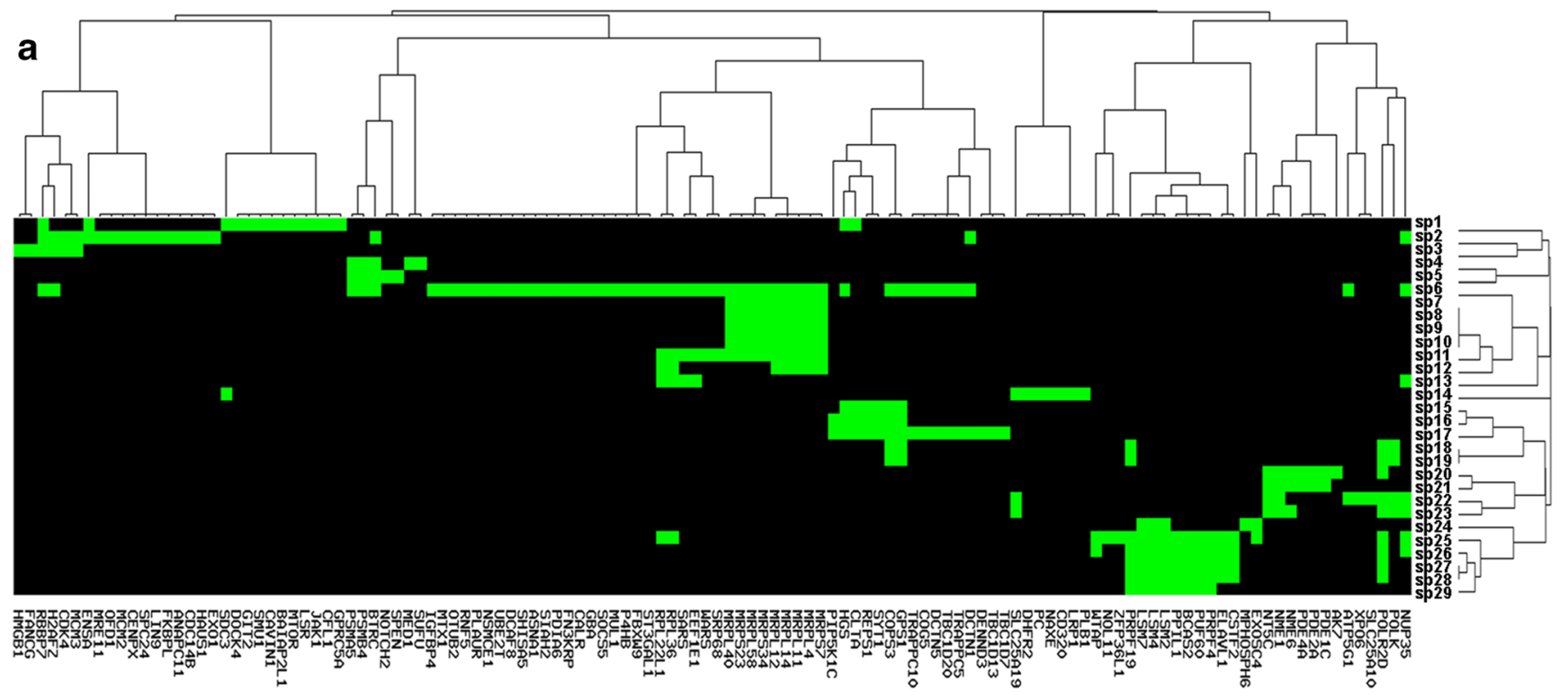

b

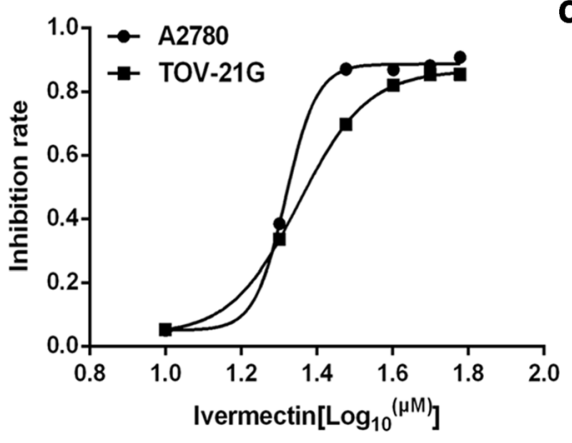

d

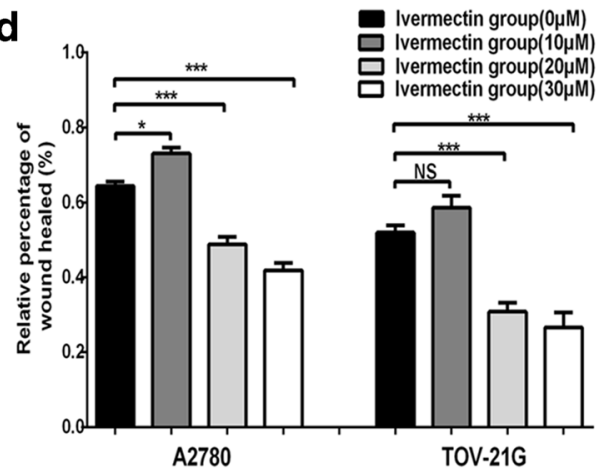

Fig. 1 Signaling pathways enriched with EIF4A3-binding mRNAs and effects of ivermectin on ovarian cancer cell migration. a Signaling pathways enriched with EIF4A3-binding mRNAs were clustered into 8 groups. $\mathrm{sp}=$ signaling pathway. $\mathrm{sp} 1=$ EGFR1; $\mathrm{sp} 2=$ Cell cycle; $\mathrm{sp} 3=$ Retinoblastoma gene in cancer; sp4 = Hedgehog; sp $5=$ Notch; sp6 = Metabolism of proteins; $\mathrm{sp} 7=$ Mitochondrial translation; $\mathrm{sp} 8=$ Mitochondrial translation elongation; $\mathrm{sp} 9=$ Mitochondrial translation initiation; $\operatorname{sp} 10=$ Mitochondrial translation termination; $s p 11=$ Translation; sp12 = Ribosome; $\mathrm{sp} 13=$ Selenoamino acid metabolism; sp14 = Metabolism of proteins; sp15 = Cargo recognition for clathrinmediated endocytosis; sp16 = Clathrin-mediated endocytosis; $\mathrm{sp} 17=$ Membrane trafficking; sp18 = Nucleotide excision repair; sp19= Transcription coupled nucleotide excision repair (TC-NER); $\mathrm{sp} 20=$

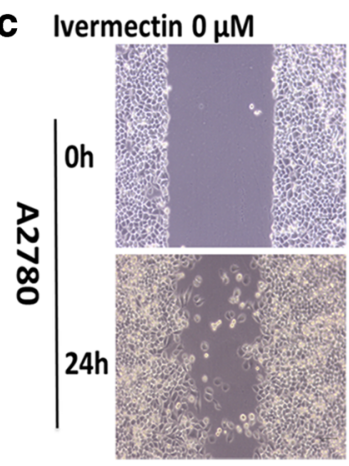

$10 \mu \mathrm{M}$

$20 \mu \mathrm{M}$

$30 \mu \mathrm{M}$
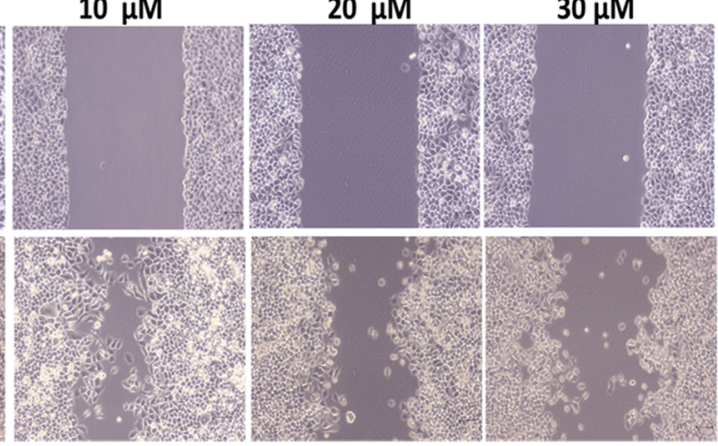

$10 \mu \mathrm{M}$

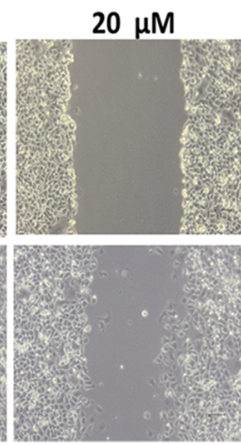

$30 \mu \mathrm{M}$

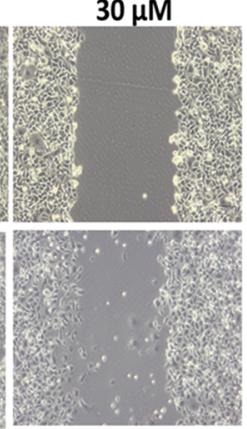

Purine metabolism; sp21 = Purine metabolism Homo sapiens (human); sp22 = Purine nucleotides nucleosides metabolism; sp23 = Pyrimidine metabolism; sp24 = RNA degradation Homo sapiens (human); sp25 = Metabolism of RNA; sp26 = Processing of capped intron-containing premRNA; sp27 = mRNA splicing; sp28 = mRNA splicing - major pathway; and sp29 $=$ Spliceosome Homo sapiens (human). b Cell viability was measured with CCK8 assay in OC cells A2780 and TOV-21G treated with ivermectin $(0-60 \mu \mathrm{M})$ for $24 \mathrm{~h}\left(n=3 ; \mathrm{X}=\log { }^{\text {(ivermectin concentration) }}\right)$. c Wound healing test of OC cells A2780 and TOV-21G treated with ivermectin $(0-30 \mu \mathrm{M})$ for $24 \mathrm{~h}(n=3)$. d Histogram statistics of wound healing test of OC cells A2780 and TOV-21G treated with ivermectin (0$30 \mu \mathrm{M})(n=3) . * p<0.05 ; * * p<0.01 ; * * * p<0.001$ 
Fig. 2 LncRNA expression profile in cells A2780 treated with ivermectin $(0-30 \mu \mathrm{M}) . * p<0.05$; $* * p<0.01 ; * * * p<0.001$
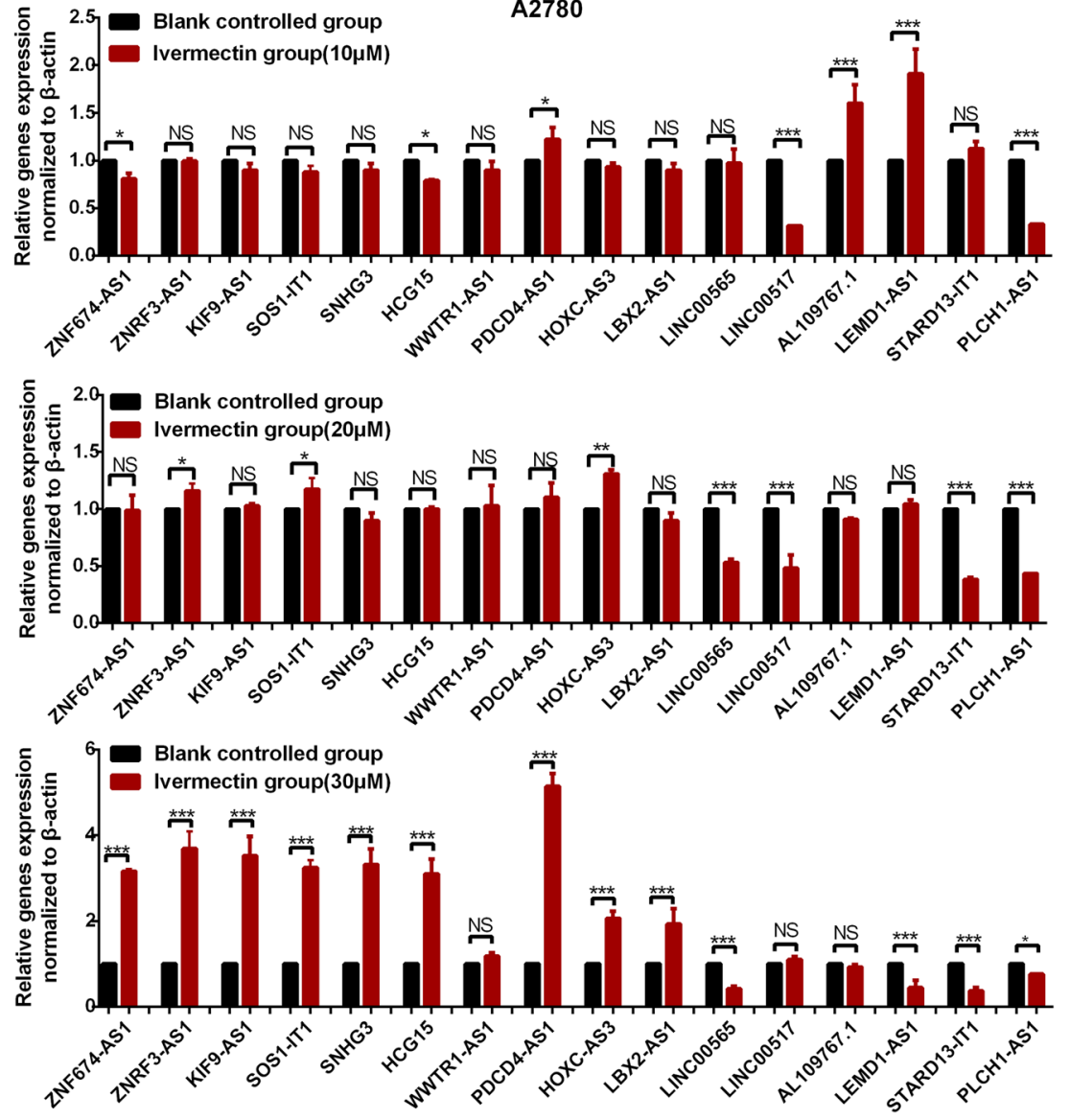

95\% CI 1.01-1.04; $p=3.70 \mathrm{E}-03$ ), cancer status (HR 10.22; 95\% CI $3.20-32.63 ; p=8.61 \mathrm{E}-05)$, primary therapy outcome success (HR 1.48; 95\% CI 1.26-1.73; $p=9.75 \mathrm{E}-07$ ), and risk score (HR 3.15; 95\% CI 1.11-8.96; $p=3.10 \mathrm{E}-03$ ) were significantly correlated with a poor OS (Fig. 7b). The risk score factor based on optimized prognostic model of three-gene signature was statistically significant at both univariate and multivariate analyses.

\section{Discussion}

Anti-parasite drug ivermectin used a broad spectrum of disease models. Recently, ivermectin, as a promising anticancer agent, showed remarkable ability to block tumor progression [36]. For example, the oncogenic kinase PAK1 was somehow essential for tumorigenesis and growth in more than $70 \%$ human cancers, including cervical, breast, thyroid, prostate, pancreatic, lung, colon, gastric, hepatoma, melanoma, and glioma cancers. Study showed that ivermectin suppressed the growth of multiple human OC cell lines in vitro and in a xenograft mouse model by inactivating PAK1 [37]. Moreover, there were almost no adverse effects for ivermectin treatment. Ivermectin exerts selective toxicity and has protective effect on normal cells [38]. In fact, the study shows that IC50 ( $30.12 \mathrm{~mol} / \mathrm{L})$ of "normal" breast epithelial cell line MCF$10 \mathrm{~A}$ was much higher than breast cancer cells $(<20 \mathrm{~mol} / \mathrm{L})$, such as cancer cells MCF-7 (9.30 mol/L), MDA-MB-231 (10.48 mol/L), MDA-MB-468 (16.20 mol/L), MDA-MB$361(13.44 \mathrm{~mol} / \mathrm{L})$, MDA-MB-435 (15.10 mol/L), and HS578T (17.29 mol/L) [9]. It clearly demonstrates that in vitro and in vivo antitumor activities of ivermectin can be achieved at relatively low concentration range, while such a concentration range is safe for healthy and parasitic patients based on human pharmacokinetic studies, which was beneficial to rapid move of ivermectin into clinical trials for cancer patients. Ivermectin was also used as chemotherapeutic sensitizer of, for example, platinum that was common chemotherapeutics for OC. Antiestrogens, including tamoxifen and fulvestrant, could be the effective treatment for $\mathrm{OC}$ in cases of platinum resistant. Ivermectin sensitized OC cells to fulvestrant and tamoxifen by blocking HE4/importin-4 nuclear accumulation [39]. Additionally, the synergy was also observed between ivermectin and BCR-ABL TKIs in acute myeloid leukemia, and the ivermectin can induce cell death and chloride-dependent membrane hyperpolarization in acute myeloid leukemia cells [12]. Considering risks and costs, drug redirecting, as the identification of novel usages for existing 
Fig. 3 LncRNA expression profile in cells TOV-21G treated with ivermectin $(0-30 \mu \mathrm{M})$. $* p<0.05 ; * * p<0.01$; $* * * p<0.001$

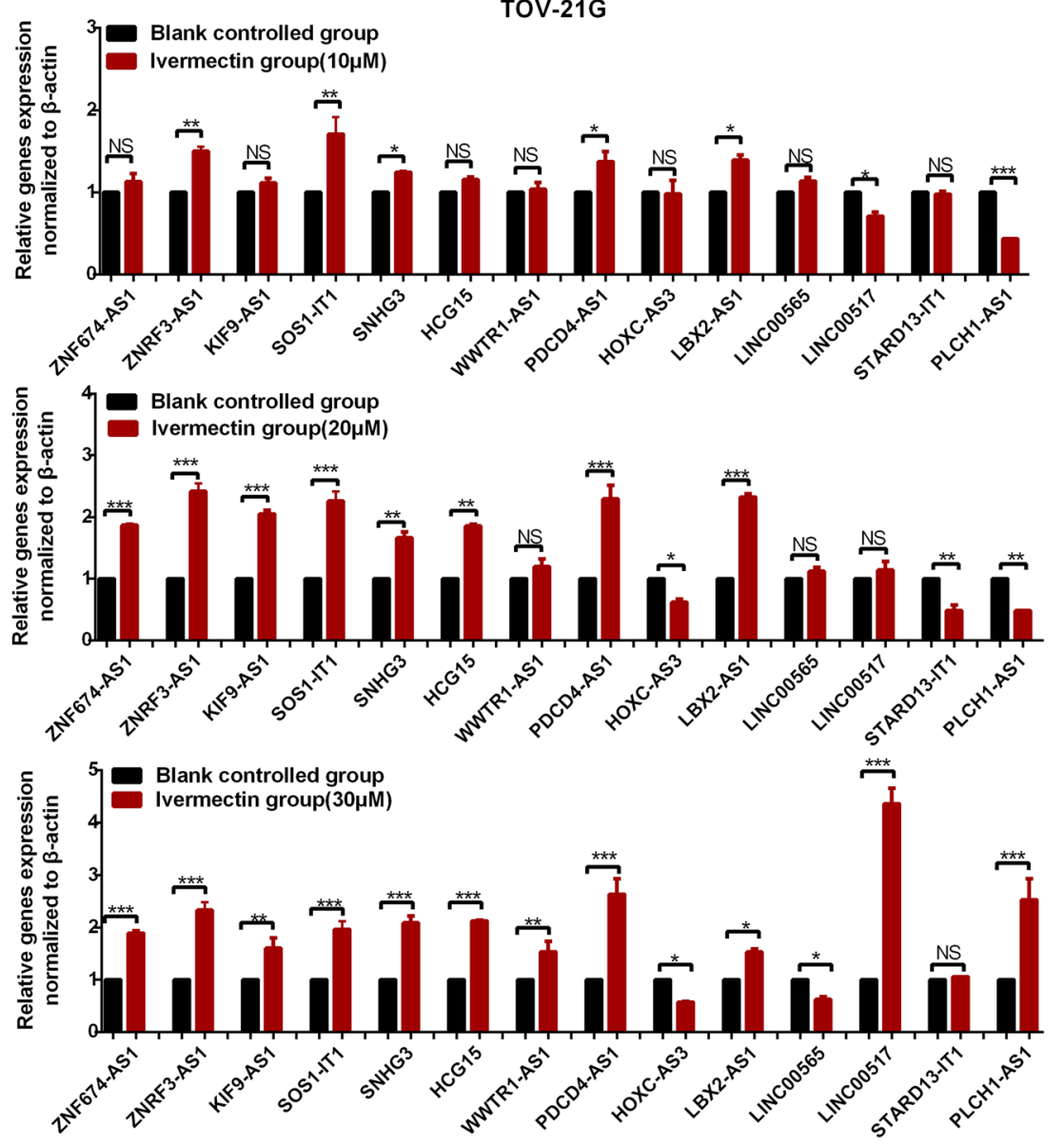

drugs, it would be possible to monitor pharmacokinetic and pharmacodynamics profiles of ivermectin in vitro and in vivo [8].

LncRNA played important roles in OC [29]. This study provided a sixteen-lnRNA signature in OCs, including HCG15, KIF9-AS1, PDCD4-AS1, ZNF674-AS1, ZNRF3AS1, SOS1-IT1, LINC00565, SNHG3, PLCH1-AS1, WWTR1-AS1, LINC00517, AL109767.1, STARD13-IT1, LBX2-AS1, LEMD1-AS1, and HOXC-AS3, which showed a changed profile when OCs were treated with ivermectin. Some of them have been proved significantly in cancers. For example, SNHG3 expressions were associated with poor prognosis and enhanced malignant progression of OC. SNHG3 was significantly increased in OC tissues compared to adjacent normal tissues. Higher SNHG3 expressions were positively associated with lymph node metastasis, OC stage, and poor prognosis [40]. RNA pull-down mass spectrometry analysis found that YBX1 interacted with HOXC-AS3, and RNA-seq analysis found a marked overlap between genes that were differentially expressed after YBX1 knockdown and those genes that were transcriptionally regulated by HOXCAS3, which suggested a novel lncRNA HOXC-AS3 mediate tumorigenesis of cancer by binding to YBX1 [41]. ZNF674AS1 expressions in the hepatocellular carcinoma cell lines
HCCLM3, HepG2, SK-Hep1, MHCC97H, Hep3B, and $\mathrm{HuH} 7$ were significantly downregulated compared to that in the normal liver cell line QSG-7701, whose results were consistent with the test of hepatocellular carcinoma tissues, and ZNF674-AS1 expressions were significantly correlated with clinical stage, metastasis, and histopathologic grading [42]. It is necessary to further study the detailed molecular mechanisms underlying ivermectin-mediated suppression of tumor growth. However, the regulatory relationships between ivermectin and lncRNAs have not been reported until now.

Our previous study found that EIF4A3 played important role in OCs, and there were various lncRNAs to interact with EIF4A3 [35]. In this study, EIF4A3 and EIF4A3-binding mRNAs were verified with SILAC-based quantitative proteomics in OC cells treated with and without ivermectin, and the signaling pathways enriched with EIF4A3-binding mRNAs were clustered into 8 groups. Some signaling pathways were closely related to cancer; for example, mitochondrial translation pathway (including initiation, elongation, and termination), mRNA splicing pathway, and Notch signaling pathway. Mitochondria can contribute to malignant transformation and tumor development through various mechanisms, such as energy metabolism, oxidative stress, cell apoptosis, dynamics, cell cycles, autophagy, and immunity process [43]. 

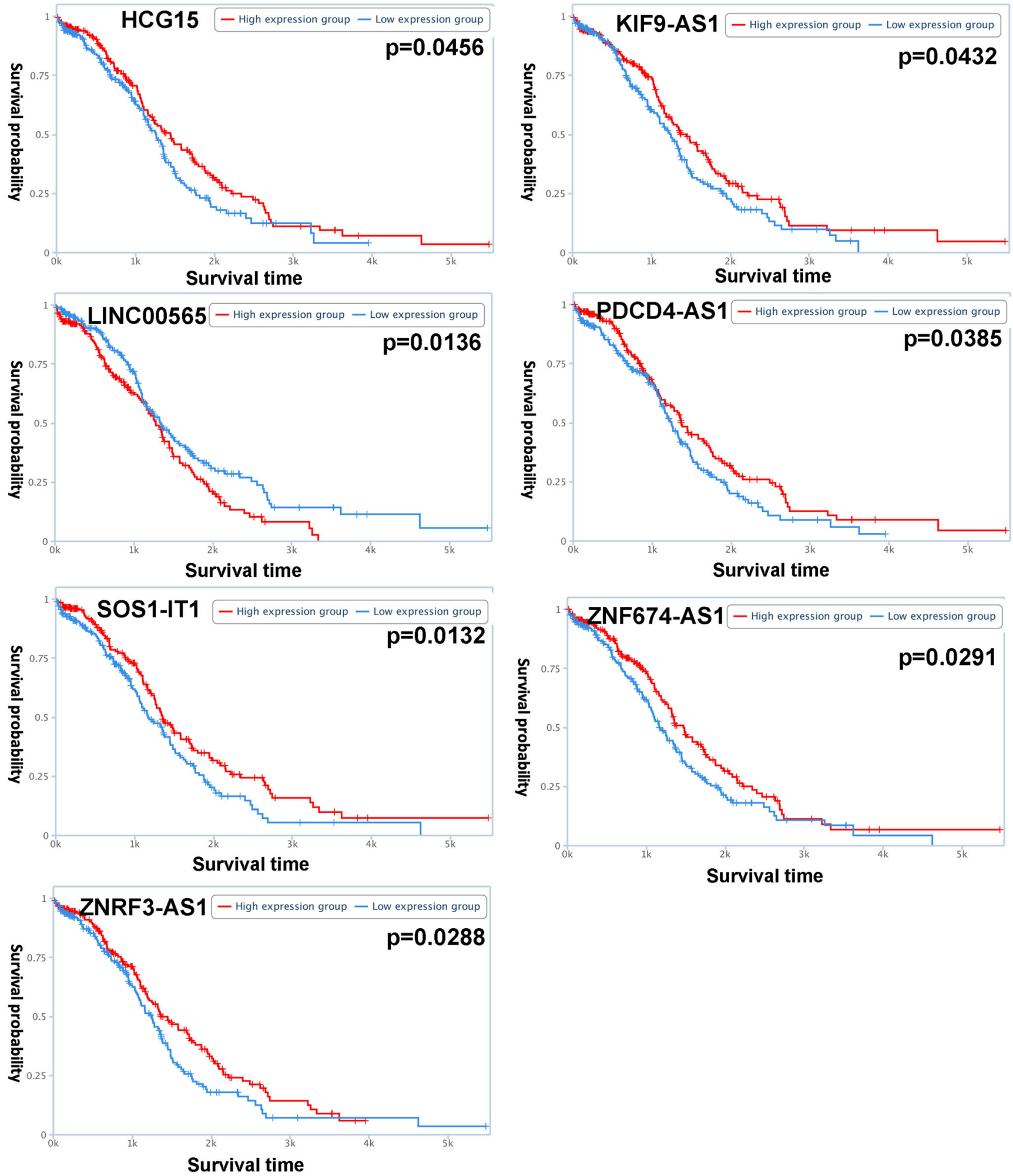

Fig. 4 Overall survival analysis of HCG15, KIF9-AS1, LINC00565, PDCD4-AS1, SOS1-IT1, ZNF674-AS1, and ZNRF3-AS1 in ovarian cancers

Mitochondria contain their own genome (also known as mtDNA) to encode two ribosomal RNAs (12S and $16 \mathrm{~S})$ in mitochondrial ribosome, 22 transfer ribonucleic acid RNAs, and 13 polypeptides. Most proteins that make up the various parts of mitochondria are encoded by nuclear genes [44].
Mitochondrial translational pathways played important role in mitochondrial protein synthesis, and those genes were identified to enrich in mitochondrial translational pathway, including MRPL11, MRPL12, MRPL14, MRPL4, MRPL40, MRPL58, MRPS23, MRPS34, and MRPS7. Some of those 

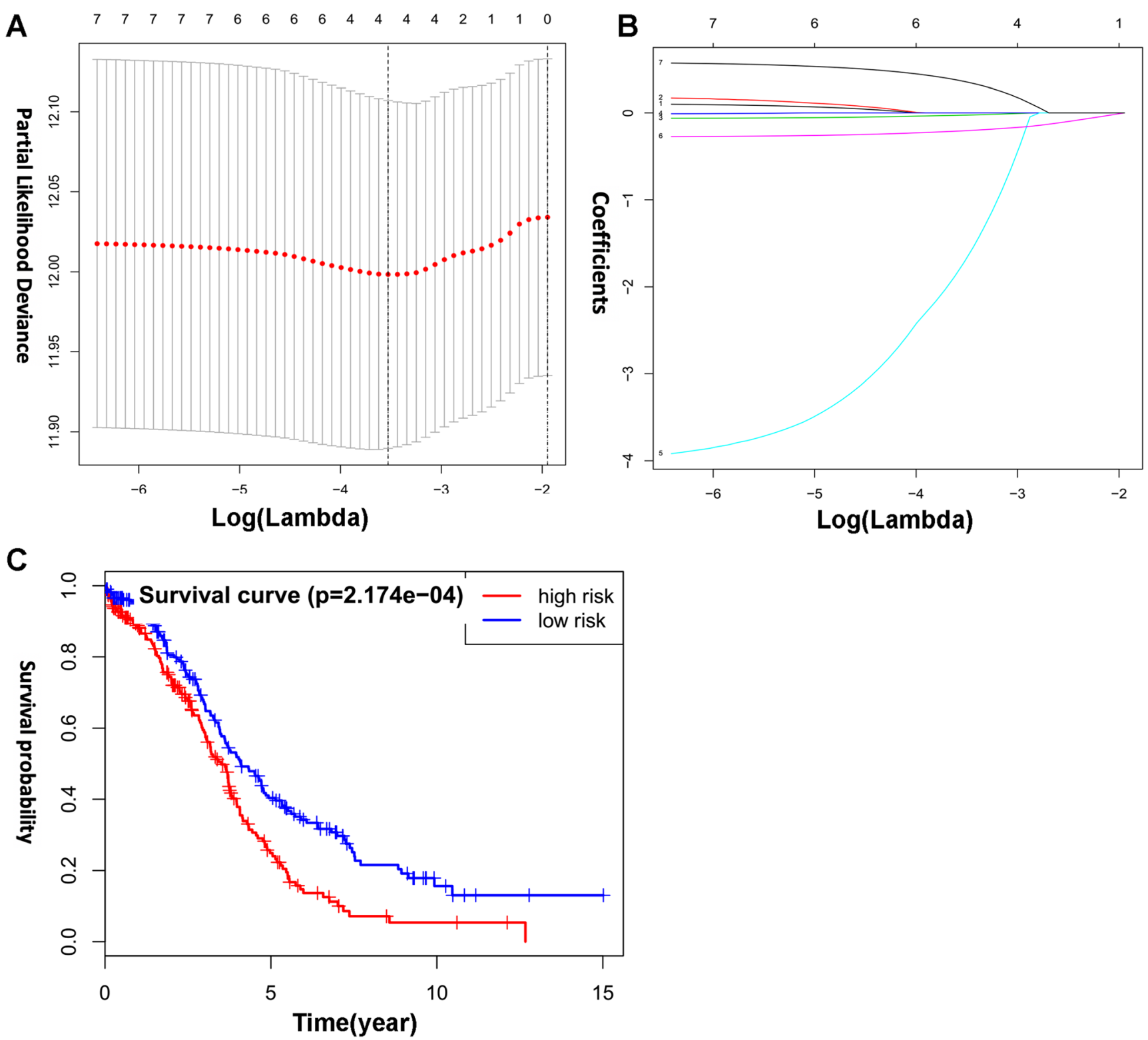

Fig. 5 Lasso regression identified the prognostic model of three-lncRNA signature. a, b Lasso regression complexity is controlled by lambda using the glmnet R package. c Overall survival analysis of three-lncRNA signature between high-risk and low-risk groups

markers of mitochondrial translation were associated with poor clinical outcome in human cancer patients. Genomewide transcriptional profiling data showed that mitochondrial ribosomal proteins were associated with mitochondrial translation upregulated in human breast cancer cells, such as MRPS7 and MRPL40 [45]. Mutations in mitochondrial ribosomal protein MRPL12 with altered integration into the large ribosomal subunit led to mitochondrial translation deficiency [46]. It would be possible to develop new anticancer therapies for targeting mitochondrial translation in human cancer cells. This study also found that the mRNA splicing pathway provided one of major mechanisms for protein diversity. More and more evidence indicated the close association of alternative mRNA splicing with cancer [47]. This study identified multiple genes enriched in mRNA splicing pathway, including BCAS2, CSTF2, ELAVL1, LSM2, LSM4, LSM7, POLR2D, PPIL1, PRPF19, PRPF4, and PUF60. PUF60 is a splicing factor that binds uridine (U)-rich tracts and facilitates association of the U2 small nuclear ribonucleoprotein with primary transcripts. For example, PUF60-activated exons uncover altered 3' splice-site selection by germline missense mutations in a single-RNA recognition motif [48]. ELAVL1 (also known as HuR) is highly expressed in different kinds of cancers, which could be useful in cancer auxiliary diagnosis, prognosis monitoring, and targeted therapy. The gene profiling analysis identified that ELAVL1 overexpression was associated with hundreds of differentially expressed genes and tens of alternative splicing events [49]. Deregulated Notch 


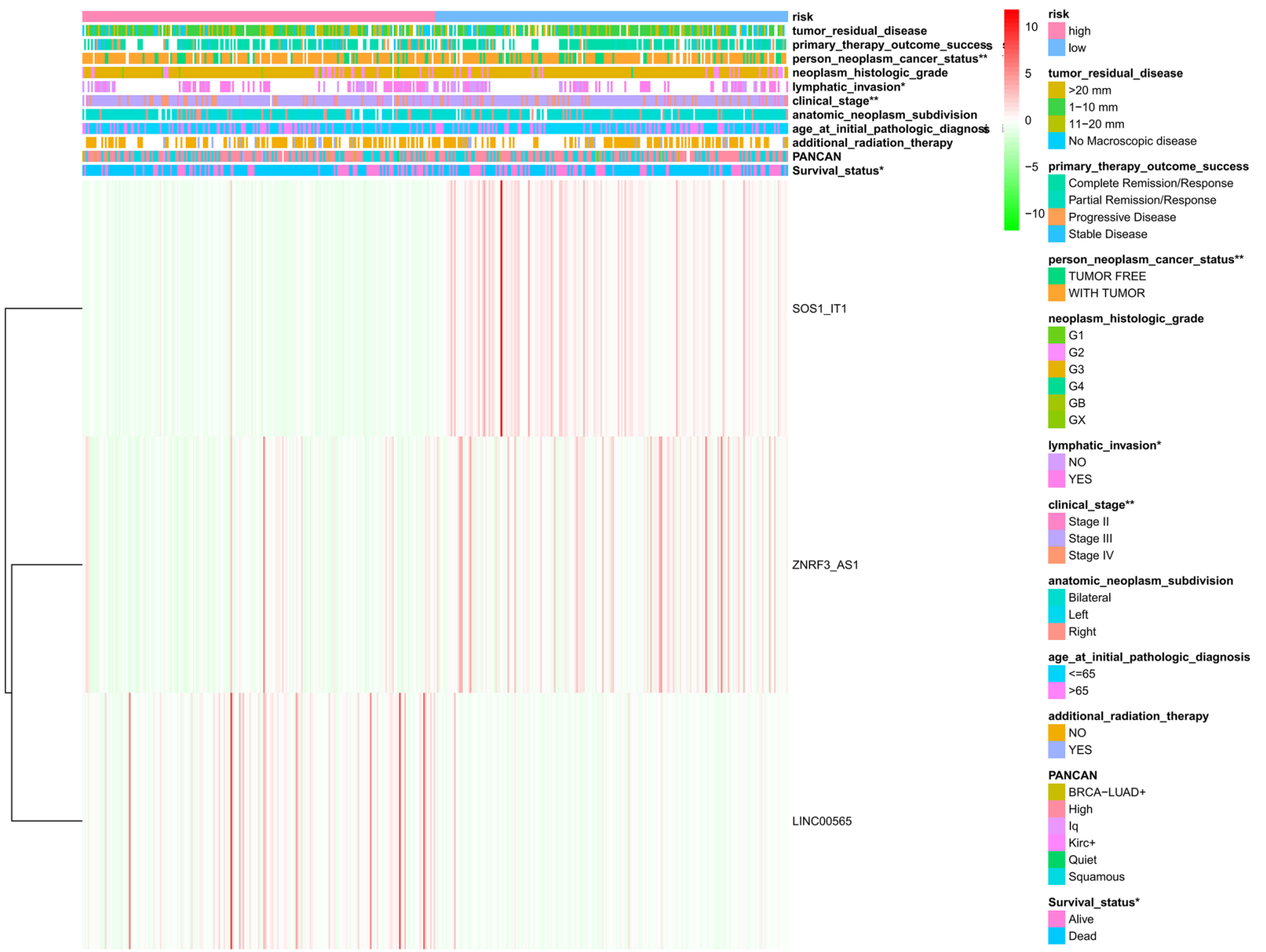

Fig. 6 The heatmap of sample risk groups and the related clinical characteristics in ovarian cancers. The clinical characteristics include survival status, PANCAN, additional radiation therapy, age at initial pathologic diagnosis, anatomic neoplasm subdivision, clinical stage,

signaling was a crucial factor to facilitate proliferation and progression of cancer cells. Four subtypes of Notch receptors have been found to play distinct roles in oncogenesis. Disrupting Notch2 signaling might yield novel cancer treatment regimens [50]. This study identified multiple genes to enrich in Notch2 signaling pathway, including BTRC, Notch2, PSMA5, PSMB4, and SPEN. The study found a small Notch2-high expression cell population existed in primary and bone metastatic breast cancers, which had a survival advantage over Notch2-low expression cell population [51]. BTRC was overexpressed in many tumors and regulated various cellular processes through degrading important targets [52]. Those previous studies were consistent with our findings that EIF4A3 played an important role in cancer through various cancer-related pathways.

The previous study found that EIF4A3 in combination with lncRNAs obstructed the recruitment of EIF4A3 to the cell cycle gene mRNA, which promoted the cancer cell lymphatic invasion, neoplasm histologic grade, cancer status, primary therapy outcome success, and tumor residual disease. $* p<0.05$; $* * p<0.01 ; * * * p<0.001$

proliferation [53]. In this study, E IF4A3 was predicted to have binding sites with 16 lncRNAs (HCG15, KIF9-AS1, PDCD4-AS1, ZNF674-AS1, ZNRF3-AS1, SOS1-IT1, LINC00565, SNHG3, PLCH1-AS1, WWTR1-AS1, LINC00517, AL109767.1, STARD13-IT1, LBX2-AS1, LEMD1-AS1, and HOXC-AS3), which were enriched in various cancer-related pathways. All findings showed that IncRNA-EIF4A3-cancer-related pathway axis was one of the potential mechanisms in the treatment of OCs. Additionally, the expressions of those 16 lncRNAs were verified by qRT-PCR before and after ivermectin treatment, which is significant to explore ivermectin-mediated lncRNA-EIF4A3-mRNA axes in OCs. The lasso regression identified an optimized three-lncRNA signature model (ZNRF3-AS1, SOS1-IT1, and LINC00565). OC is a multifaceted disease that was involved in multifactor, complex biological processes, and unpredictable consequences. It is not reasonable to use a single molecule as biomarker for 


$\begin{array}{lcr}\text { A } & \text { pvalue } & \text { Hazard ratio } \\ \text { PANCAN } & 0.029 & 0.909(0.835-0.990) \\ \text { additional_radiation_therapy } & 0.334 & 0.794(0.497-1.268) \\ \text { age_at_initial_pathologic_diagnosis } & <0.001 & 1.022(1.010-1.034) \\ \text { anatomic_neoplasm_subdivision } & 0.023 & 1.345(1.042-1.736) \\ \text { clinical_stage } & 0.058 & 1.301(0.991-1.708) \\ \text { lymphatic_invasion } & 0.561 & 1.154(0.712-1.869) \\ \text { neoplasm_histologic_grade } & & 1.126(0.853-1.486) \\ \text { person_neoplasm_cancer_status } & <0.001 & 10.957(5.127-23.417) \\ \text { primary_therapy_outcome_success } & <0.001 & 1.552(1.354-1.780) \\ \text { tumor_residual_disease } & <0.001 & 1.293(1.122-1.489) \\ & <0.001 & 3.091(1.846-5.174)\end{array}$

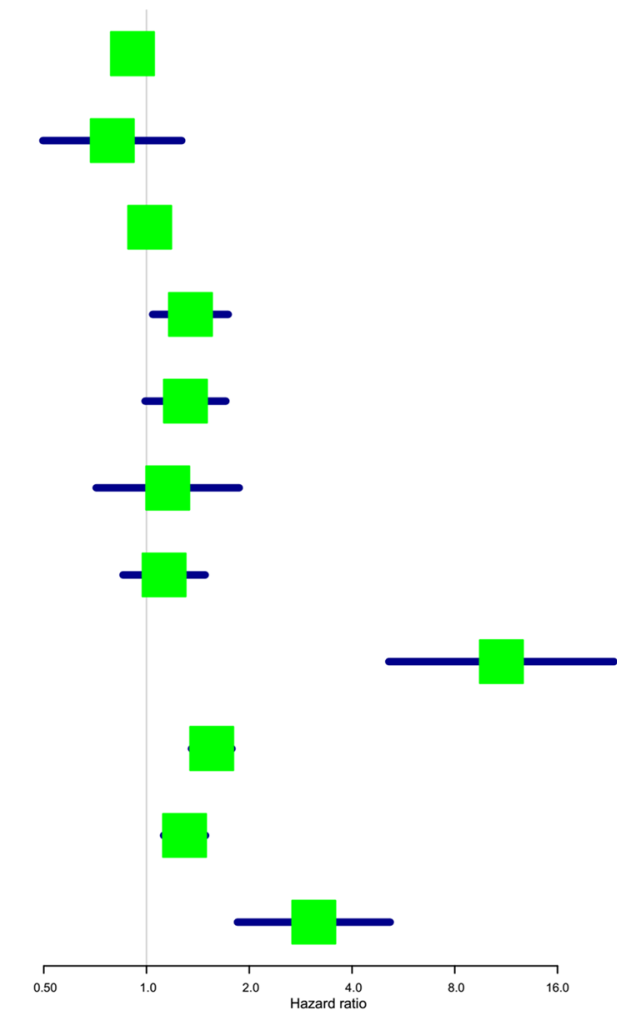

B

pvalue

Hazard ratio

PANCAN

0.280

$0.942(0.844-1.050)$

age_at_initial_pathologic_diagnosis

0.004

$1.023(1.007-1.039)$

anatomic_neoplasm_subdivision

0.324

$1.167(0.859-1.585)$

person_neoplasm_cancer_status

$<0.001$

10.224(3.204-32.625)

primary_therapy_outcome_success

$<0.001$

$1.478(1.264-1.728)$

tumor_residual_disease

0.792

$0.975(0.809-1.176)$

risk_score

0.031

$3.154(1.110-8.961)$

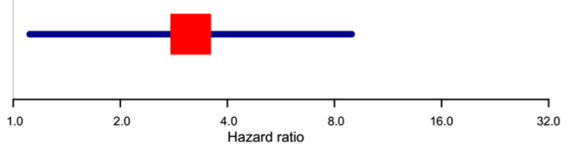

Fig. 7 The univariate (a) and multivariate (b) analyses of risk factors in ovarian cancers 
accurate predictive, preventive, and personalized medicine (PPPM) practice [22]. Some aspects of predictive medicine approach have been reported in breast cancer associated with tumorigenesis, metastasis, women health according to patient's age, sex, and phenotype [54-56]. Individualized patient profiles, risks, and mitigating measures would be of great help for the development of PPPM [57]. In this study, those three lncRNAs in the three-lncRNA signature model were never studied in OCs and had positive responses to treatment of ivermectin. The heatmap identified the relationship between the expression profile of those three lncRNAs and the OC phenotypes based on RNA sequencing in different samples. Based on the expression levels of ZNRF3-AS1, SOS1-IT1, and LINC00565, OC patients were divided into high-risk and low-risk groups, which were significantly related to cancer status, clinical stage, lymphatic invasion, and survival status. Ivermectin could mediate lncRNAs, including ZNRF3-AS1, SOS1-IT1, and LINC00565, to affect growth and metastasis of OCs. Those indicated that specific properties of phenotype according to patient's cancer status, clinical stage, lymphatic invasion, and survival status might be potentially associated with ivermectin-related moleculepanel.

\section{Strength and limitation}

lncRNA-EIF4A3-mRNA axes functioned in ivermectinmeditated suppression of OC with a good reproducibility, which is supported with the following evidence: (i) lncRNA-EIF4A3-mRNA axes exist in OC [32] and glioma [33], and some drugs can target these axes to generate antitumor effects in epithelial OC cells [32]. (ii) Our previous study found that lncRNA SNHG3-EIF4A3-energy metabolic pathways were important for OCs [35]. (iii) This study found that the expressions of lncRNAs (SNHG3, HCG15, KIF9-AS1, PDCD4-AS1, ZNF674-AS1, ZNRF3-AS1, SOS1-IT1, LINC00565, PLCH1-AS1, WWTR1-AS1, LINC00517, AL109767.1, STARD13-IT1, LBX2-AS1, LEMD1-AS1, and HOXC-AS3) in cells TOV-21G and A2780 were significantly changed before and after treatment with ivermectin $(0 \mu \mathrm{M}, 10 \mu \mathrm{M}, 20 \mu \mathrm{M}$, and $30 \mu \mathrm{M})$. (iv) SILAC-based quantitative proteomics found ivermectin significantly downregulated the protein expression levels of EIF4A3 and 116 EIF4A3-binding mRNAs (Table 2). (v) TCGA transcriptomics data-based bioinformatics revealed the binding relationships between 16 lncRNAs and EIF4A3 and between EIF4A3 and EIF4A3-binding mRNAs [35]. Thereby, it has important scientific value to explore ivermectin-mediated lncRNA-EIF4A3-mRNA axes in OC. Clarification of these lncRNA-EIF4A3-mRNA axes for molecular mechanism of ivermectin-mediated suppression of OC would significantly contribute to personalized drug therapy for OC and discovery of effective and reliable ivermectin-mediated molecule-panel biomarkers for predictive diagnosis and prognostic assessment. Another strength of this study is that we optimized and established a threelncRNAs signature based on the ivemectin-mediated IncRNA-EIF4A3-mRNA axes, which is potential lncRNApanel biomarker for OC survival prediction to predict patient's cancer status, clinical stage, lymphatic invasion, and survival status.

However, one must realize the limitations remaining in the current study. (i) The binding sites between EIF4A3 and 16 lncRNAs and between EIF4A3 and EIF4A3binding candidate mRNAs should be further verified. An RNA-binding protein immunoprecipitation assay in OC cell lines would be an appropriate approach to resolve this scientific issue. Clarification of these EIF4A3-binding sites might benefit for in-depth understanding of the molecular mechanism of ivermectin and the new personalized therapeutic targets for OC. (ii) Two cell lines (TOV-21G and A2780) used in this current study cannot cover all characteristics of OC. It is necessary to analyze more OC cells for revealing the individualized and common characteristic-related effects of ivermectin in treatment of OC. (iii) Except for TCGA database, other cohort studies would be needed for further validation of ivermectin-related three-lncRNA-signature model for OC prognosis and predictive diagnostics. (iv) Large-scale clinical data are also needed to validate ivermectin-related lncRNA-panel biomarker for OC survival prediction after the treatment with ivermectin. Moreover, although ivermectin is extensively used for anti-parasites, currently, its antitumor effects are all derived from the experimental studies in the cell and animal models. It has not been used in cancer patients for clinical trials. Thus, extensive studies will still be needed for the realistic implementation of ivermectin in individual or in combination with other drugs into clinical practice in prediction, prevention, and personalized drug therapy of OCs.

\section{Future studies}

Considering strengths and limitations of the currently presented study discussed in the context of 3PM, further studies are proposed here to maximize the potentials of ivermectinmediated lncRNA-EIF4A3-mRNA axes for PPPM in OC. (i) RNA-binding protein immunoprecipitation assay will be carried out in OCs to validate the binding relationship between EIF4A3 and 16 lncRNAs, and between EIF4A3 and 116 EIF4A3-binding mRNAs, and further experimentally define those accurate binding sites. (ii) More OC cell lines will be analyzed to expand and validate the link of ivermectin and EIF4A3 and observe how this link regulates IncRNA expressions and targeted mRNA expressions, and then define the phenotype of OCs. (iii) More clinical samples will be collected to discover ivermectin-related lncRNA-panel 
biomarker for OC towards its PPPM. (iv) Focusing on the ivemectin-mediated lncRNA-EIF4A3-mRNA axes, one might design and discover multiple therapeutic targets for multi-target drug therapy to synergize and amplify the therapeutic effect of ivermectin towards its personalized drug therapy for OCs. (v) Currently, no evidence supports that ivermectin can prevent the occurrence of OC. However, ivermectin is an extensively used anti-parasite drug in normal people without OC, and it has anticancer effects. Can ivemectin prevent the occurrence of $\mathrm{OC}$ and even other cancers? It would be an interesting scientific problem. First, we may design animal models to observe whether ivermectin can prevent the occurrence of OCs and further design a large-scale and multicenter cohort study. It will directly contribute to the preventive medicine strategy for OC.

\section{Conclusions}

This study found that ivermectin inhibited OC migration, and that ivermectin-mediated lncRNA-EIF4A3-mRNA axes were the potential mechanism in OCs. The optimized threelncRNA signature model (ZNRF3-AS1, SOS1-IT1, and LINC00565) provided a good assessment system to significantly associate survival risk with the expression profile of these three lncRNAs. These findings are the precious resource and have practical significance for drug redirecting of ivermectin in OC treatment for personalized drug therapy and prognostic assessment towards its PPPM practice.

\section{Endnotes and recommendation in regard to PPPM}

We recommend to strengthen the study on relationship of IncRNA and drug to understand in depth the molecular mechanism of ivermectin-depressing OC and the study on integration of lncRNAs and large-scale clinical data to discover ivermectin-related lncRNA-panel biomarker for OC survival prediction treated with ivermectin. The IncRNA-EIF4A3mRNA axes play important roles in the therapeutic process of ivermectin treatment of OC. EIF4A3 is a central molecule in the ivermectin drug signaling pathway to realize the personalized drug therapy of ivermectin in OC. Moreover, it is well known that the development of an anticancer new drug is a time-consuming long-term research process and needs lots of money, and the huge increase in national healthcare costs is becoming a concern worldwide. In such a social context, the repositioning of the existed drugs for their new uses for other diseases has recently gained extensive attentions. Ivermactin as a common used anti-parasite drug has been found to have anticancer effects, which will play an important role in future drug repositioning strategies for OC [58]. The personalized drug therapy of ivermectin would be a primary concrete practice for PPPM according to conceptual PPPM papers [59, 60].
Authors' contributions N.L. performed bioinformatic analysis, carried out qRT-PCR experiments, prepared figures and tables, and designed and wrote the manuscript. X.Z. conceived the concept, instructed experiments and data analysis, supervised results, coordinated, critically revised/wrote manuscript, and was responsible for its financial supports and the corresponding works. All the authors approved the final manuscript.

Funding information The authors received financial supports from the Shandong First Medical University Talent Introduction Funds (to X.Z.), the Hunan Provincial Hundred Talent Plan (to X.Z.), and Central South University Graduate Student Exploration Innovative Project 2019 (Grant No. 206021701).

\section{Compliance with ethical standards}

Competing interests The authors declare that they have no competing interests.

Consent publication Not applicable.

Ethical approval Not applicable.

\section{References}

1. Campbell WC. History of avermectin and ivermectin, with notes on the history of other macrocyclic lactone antiparasitic agents. Curr Pharm Biotechnol. 2012;13:853-65. https://doi.org/10.2174/ 138920112800399095.

2. Crump A. Ivermectin: enigmatic multifaceted 'wonder' drug continues to surprise and exceed expectations. J Antibiot (Tokyo). 2017;70:495-505. https://doi.org/10.1038/ja.2017.11.

3. Diao H, Cheng N, Zhao Y, Xu H, Dong H, Thamm DH, et al. Ivermectin inhibits canine mammary tumor growth by regulating cell cycle progression and WNT signaling. BMC Vet Res. 2019;15: 276. https://doi.org/10.1186/s12917-019-2026-2.

4. Gallardo F, Mariame B, Gence R, Tilkin-Mariame AF. Macrocyclic lactones inhibit nasopharyngeal carcinoma cells proliferation through PAK1 inhibition and reduce in vivo tumor growth. Drug Des Devel Ther. 2018;12:2805-14. https://doi.org/10.2147/dddt. s172538.

5. Markowska A, Kaysiewicz J, Markowska J, Huczynski A. Doxycycline, salinomycin, monensin and ivermectin repositioned as cancer drugs. BMC Vet Res. 2019;29:1549-54. https://doi.org/ 10.1016/j.bmcl.2019.04.045.

6. Patel BC, Ostwal S, Sanghavi PR, Joshi G, Singh R. Management of malignant wound myiasis with ivermectin, albendazole, and clindamycin (triple therapy) in advanced head-and-neck cancer patients: a prospective observational study. Indian J Palliat Care. 2018;24:459-64. https://doi.org/10.4103/ijpc.ijpc_112_18.

7. Ferenc NN, Levin M. Effects of ivermectin exposure on regeneration of D. dorotocephala planaria: Exploiting human-approved ion channel drugs as morphoceuticals. Macromol Biosci. 2019;19: e1800237. https://doi.org/10.1002/mabi.201800237.

8. Juarez M, Schcolnik-Cabrera A, Duenas-Gonzalez A. The multitargeted drug ivermectin: from an antiparasitic agent to a repositioned cancer drug. Am J Cancer Res. 2018;8:317-31.

9. Dou Q, Chen HN, Wang K, Yuan K, Lei Y, Li K, et al. Ivermectin induces cytostatic autophagy by blocking the PAK1/Akt axis in breast cancer. Cancer Res. 2016;76:4457-69. https://doi.org/10. 1158/0008-5472.can-15-2887. 
10. Mate L, Ballent M, Canton C, Ceballos L, Lifschitz A, Lanusse C, et al. Assessment of P-glycoprotein gene expression in adult stage of Haemonchus contortus in vivo exposed to ivermectin. Vet Parasitol. 2018;264:1-7. https://doi.org/10.1016/j.vetpar.2018. 10.011.

11. Raza A, Kopp SR, Kotze AC. Synergism between ivermectin and the tyrosine kinase/P-glycoprotein inhibitor crizotinib against Haemonchus contortus larvae in vitro. Vet Parasitol. 2016;227: 64-8. https://doi.org/10.1016/j.vetpar.2016.07.026.

12. Sharmeen S, Skrtic M, Sukhai MA, Hurren R, Gronda M, Wang X, et al. The antiparasitic agent ivermectin induces chloride-dependent membrane hyperpolarization and cell death in leukemia cells. Blood. 2010;116:3593-603. https://doi.org/10.1182/blood-2010$01-262675$.

13. Wang J, Xu Y, Wan $\mathrm{H}, \mathrm{Hu}$ J. Antibiotic ivermectin selectively induces apoptosis in chronic myeloid leukemia through inducing mitochondrial dysfunction and oxidative stress. Biochem Biophys Res Commun. 2018;497:241-7. https://doi.org/10.1016/j.bbrc. 2018.02.063.

14. Zhu M, Yancy HF, Deaver C, Jones YL, Myers MJ. Loperamideinduced expression of immune and inflammatory genes in collies associated with ivermectin sensitivity. J Vet Pharmacol Ther. 2016;39:131-7. https://doi.org/10.1111/jvp.12268.

15. Dominguez-Gomez G, Chavez-Blanco A, Medina-Franco JL, Saldivar-Gonzalez F, Flores-Torrontegui Y, Juarez M, et al. Ivermectin as an inhibitor of cancer stemlike cells. Mol Med Rep. 2018;17:3397-403. https://doi.org/10.3892/mmr.2017.8231.

16. Yin J, Park G, Lee JE, Choi EY, Park JY, Kim TH, et al. DEADbox RNA helicase DDX23 modulates glioma malignancy via elevating miR-21 biogenesis. Brain. 2015;138:2553-70. https://doi. org/10.1093/brain/awv167.

17. Munoz J, Ballester MR, Antonijoan RM, Gich I, Rodriguez M, Colli E, et al. Safety and pharmacokinetic profile of fixed-dose ivermectin with an innovative $18 \mathrm{mg}$ tablet in healthy adult volunteers. PLoS Negl Trop Dis. 2018;12:e0006020. https://doi.org/10. 1371/journal.pntd.0006020.

18. Zhang X, Qin T, Zhu Z, Hong F, Xu Y, Zhang X, et al. Ivermectin augments the in vitro and in vivo efficacy of cisplatin in epithelial ovarian cancer by suppressing Akt/mTOR signaling. Am J Med Sci. 2020;359:123-9. https://doi.org/10.1016/j.amjms.2019.11. 001.

19. Golubnitschaja O, Costigliola V, EPMA. General report \& recommendations in predictive, preventive and personalised medicine 2012: white paper of the European Association for Predictive, Preventive and Personalised Medicine. EPMA J. 2012;3:14. https://doi.org/10.1186/1878-5085-3-14.

20. Hu R, Wang X, Zhan X. Multi-parameter systematic strategies for predictive, preventive and personalised medicine in cancer. EPMA J. 2013;4:2. https://doi.org/10.1186/1878-5085-4-2.

21. Cheng T, Zhan X. Pattern recognition for predictive, preventive, and personalized medicine in cancer. EPMA J. 2017;8:51-60. https://doi.org/10.1007/s13167-017-0083-9.

22. Lu M, Zhan X. The crucial role of multiomic approach in cancer research and clinically relevant outcomes. EPMA J. 2018;9:77102. https://doi.org/10.1007/s13167-018-0128-8.

23. Lu M, Wang Y, Zhan X. The MAPK pathway-based drug therapeutic targets in pituitary adenomas. Front Endocrinol (Lausanne). 2019;10:330. https://doi.org/10.3389/fendo.2019.00330.

24. Zhan X, Long Y. Exploration of molecular network variations in different subtypes of human non-functional pituitary adenomas. Front Endocrinol (Lausanne). 2016;7:13. https://doi.org/10.3389/ fendo.2016.00013.

25. Cui JJ, Wang LY, Tan ZR, Zhou HH, Zhan X, Yin JY. Mass spectrometry-based personalized drug therapy. Mass Spectrom Rev. 2020. https://doi.org/10.1002/mas.21620.
26. Renganathan A, Felley-Bosco E. Long noncoding RNAs in cancer and therapeutic potential. Adv Exp Med Biol. 2017;1008:199-222. https://doi.org/10.1007/978-981-10-5203-3_7.

27. Lee JT, Bartolomei MS. X-inactivation, imprinting, and long noncoding RNAs in health and disease. Cell. 2013;152:1308-23. https://doi.org/10.1016/j.cell.2013.02.016.

28. Zhao J, Li L, Han ZY, Wang ZX, Qin LX. Long noncoding RNAs, emerging and versatile regulators of tumor-induced angiogenesis. Am J Cancer Res. 2019;9:1367-81.

29. Li N, Zhan X. Identification of clinical trait-related lncRNA and mRNA biomarkers with weighted gene co-expression network analysis as useful tool for personalized medicine in ovarian cancer. EPMA J. 2019;10:273-90. https://doi.org/10.1007/s13167-01900175-0.

30. Akhade VS, Pal D, Kanduri C. Long noncoding RNA: genome organization and mechanism of action. Adv Exp Med Biol. 2017;1008:47-74. https://doi.org/10.1007/978-981-10-5203-3 2.

31. Lin Y, Zhang J, Cai J, Liang R, Chen G, Qin G, et al. Systematic analysis of gene expression alteration and co-expression network of eukaryotic initiation factor 4A-3 in cancer. J Cancer. 2018;9:456877. https://doi.org/10.7150/jca.27655.

32. Zhang S, Leng T, Zhang Q, Zhao Q, Nie X, Yang L. Sanguinarine inhibits epithelial ovarian cancer development via regulating long non-coding RNA CASC2-EIF4A3 axis and/or inhibiting NFkappaB signaling or PI3K/AKT/mTOR pathway. Biomed Pharmacother. 2018;102:302-8. https://doi.org/10.1016/j.biopha. 2018.03.071.

33. Tang W, Wang D, Shao L, Liu X, Zheng J, Xue Y, et al. LINC00680 and TTN-AS1 stabilized by EIF4A3 promoted malignant biological behaviors of glioblastoma cells. Mol Ther Nucleic Acids. 2020;19:905-21. https://doi.org/10.1016/j.omtn.2019.10. 043.

34. Li N, Li H, Cao L, Zhan X. Quantitative analysis of the mitochondrial proteome in human ovarian carcinomas. Endocr Relat Cancer. 2018;25:909-31. https://doi.org/10.1530/erc-18-0243.

35. Li N, Zhan X, Zhan X. The lncRNA SNHG3 regulates energy metabolism of ovarian cancer by an analysis of mitochondrial proteomes. Gynecol Oncol. 2018;150:343-54. https://doi.org/10. 1016/j.ygyno.2018.06.013.

36. Laing R, Gillan V, Devaney E. Ivermectin - old drug, new tricks? Trends Parasitol. 2017;33:463-72. https://doi.org/10.1016/j.pt. 2017.02.004.

37. Hashimoto H, Sudo T, Maruta H, Nishimura R. The direct PAK1 inhibitor, TAT-PAK18, blocks preferentially the growth of human ovarian cancer cell lines in which PAK1 is abnormally activated by autophosphorylation at Thr 423. Drug Discov Ther. 2010;4:1-4.

38. Wang K, Gao W, Dou Q, Chen H, Li Q, Nice EC, et al. Ivermectin induces PAK1-mediated cytostatic autophagy in breast cancer. Autophagy. 2016;12:2498-9. https://doi.org/10.1080/15548627. 2016.1231494.

39. Lokich E, Singh RK, Han A, Romano N, Yano N, Kim K, et al. HE4 expression is associated with hormonal elements and mediated by importin-dependent nuclear translocation. Sci Rep. 2014;4: 5500. https://doi.org/10.1038/srep05500.

40. Hong L, Chen W, Wu D, Wang Y. Upregulation of SNHG3 expression associated with poor prognosis and enhances malignant progression of ovarian cancer. Cancer Biomark. 2018;22:367-74. https://doi.org/10.3233/cbm-170710.

41. Zhang E, He X, Zhang C, Su J, Lu X, Si X, et al. A novel long noncoding RNA HOXC-AS3 mediates tumorigenesis of gastric cancer by binding to YBX1. Genome Biol. 2018;19:154. https:// doi.org/10.1186/s13059-018-1523-0.

42. Zhang L, He T, Yan Y, Zhang Y, Zhou X, Huang P, et al. Expression and clinical significance of the novel long noncoding RNA ZNF674-AS1 in human hepatocellular carcinoma. Biomed 
Res Int. 2016;2016:3608914-5. https://doi.org/10.1155/2016/ 3608914.

43. Roth KG, Mambetsariev I, Kulkarni P, Salgia R. The mitochondrion as an emerging therapeutic target in cancer. Trends Mol Med. 2019;26:119-34. https://doi.org/10.1016/j.molmed.2019.06.009.

44. Baechler SA, Dalla Rosa I, Spinazzola A. Beyond the unwinding: role of TOP1MT in mitochondrial translation. Cell Cycle. 2019:18. https://doi.org/10.1080/15384101.2019.1646563.

45. Sotgia F, Whitaker-Menezes D, Martinez-Outschoorn UE, Salem AF, Tsirigos A, Lamb R, et al. Mitochondria "fuel" breast cancer metabolism: fifteen markers of mitochondrial biogenesis label epithelial cancer cells, but are excluded from adjacent stromal cells. Cell Cycle. 2012;11:4390-401. https://doi.org/10.4161/cc.22777.

46. Serre V, Rozanska A, Beinat M, Chretien D, Boddaert N, Munnich A, et al. Mutations in mitochondrial ribosomal protein MRPL12 leads to growth retardation, neurological deterioration and mitochondrial translation deficiency. Biochim Biophys Acta. 1832;2013:1304-12. https://doi.org/10.1016/j.bbadis.2013.04.014.

47. Li Y, Sun N, Lu Z, Sun S, Huang J, Chen Z, et al. Prognostic alternative mRNA splicing signature in non-small cell lung cancer. Cancer Lett. 2017;393:40-51. https://doi.org/10.1016/j.canlet. 2017.02.016.

48. Kralovicova J, Sevcikova I, Stejskalova E, Obuca M, Hiller M, Stanek D, et al. PUF60-activated exons uncover altered 3' splicesite selection by germline missense mutations in a single RRM. Nucleic Acids Res. 2018;46:6166-87. https://doi.org/10.1093/nar/ gky389.

49. Carrascoso I, Alcalde J, Tabas-Madrid D, Oliveros JC, Izquierdo JM. Transcriptome-wide analysis links the short-term expression of the $\mathrm{b}$ isoforms of TIA proteins to protective proteostasis-mediated cell quiescence response. PLoS One. 2018;13:e0208526. https:// doi.org/10.1371/journal.pone.0208526.

50. Xiu MX, Liu YM. The role of oncogenic Notch2 signaling in cancer: a novel therapeutic target. Am J Cancer Res. 2019;9:837-54.

51. Capulli M, Hristova D, Valbret Z, Carys K, Arjan R, Maurizi A, et al. Notch2 pathway mediates breast cancer cellular dormancy and mobilisation in bone and contributes to haematopoietic stem cell mimicry. Br J Cancer. 2019;121:157-71. https://doi.org/10.1038/ s41416-019-0501-y.

52. Cohen M, Amir S, Golan M, Ben-Neriah Y, Mabjeesh NJ. BetaTrCP upregulates HIF-1 in prostate cancer cells. Prostate. 2019;79: 403-13. https://doi.org/10.1002/pros.23746.
53. Han D, Gao X, Wang M, Qiao Y, Xu Y, Yang J, et al. Long noncoding RNA H19 indicates a poor prognosis of colorectal cancer and promotes tumor growth by recruiting and binding to eIF4A3. Oncotarget. 2016;7:22159-73. https://doi.org/10.18632/ oncotarget. 8063.

54. Bubnov R, Polivka J Jr, Zubor P, Konieczka K, Golubnitschaja O. "Pre-metastatic niches" in breast cancer: are they created by or prior to the tumour onset? "Flammer syndrome" relevance to address the question. EPMA J. 2017;8:141-57. https://doi.org/10.1007/ s13167-017-0092-8.

55. Zubor P, Gondova A, Polivka J Jr, Kasajova P, Konieczka K, Danko J, et al. Breast cancer and Flammer syndrome: any symptoms in common for prediction, prevention and personalised medical approach? EPMA J. 2017;8:129-40. https://doi.org/10.1007/ s13167-017-0089-3.

56. Smokovski I, Risteski M, Polivka J Jr, Zubor P, Konieczka K, Costigliola V, et al. Postmenopausal breast cancer: European challenge and innovative concepts. EPMA J. 2017;8:159-69. https:// doi.org/10.1007/s13167-017-0094-6.

57. Goncharenko V, Bubnov R, Polivka J Jr, Zubor P, Biringer K, Bielik T, et al. Vaginal dryness: individualised patient profiles, risks and mitigating measures. EPMA J. 2019;10:73-9. https://doi.org/ 10.1007/s13167-019-00164-3.

58. Kobayashi Y, Banno K, Kunitomi H, Tominaga E, Aoki D. Current state and outlook for drug repositioning anticipated in the field of ovarian cancer. J Gynecol Oncol. 2019;30:e10. https://doi.org/10. 3802/jgo.2019.30.e10.

59. Golubnitschaja O, Baban B, Boniolo G, Wang W, Bubnov R, Kapalla M, et al. Medicine in the early twenty-first century: paradigm and anticipation - EPMA position paper 2016. EPMA J. 2016;7:23. https://doi.org/10.1186/s13167-016-0072-4.

60. Grech G, Zhan X, Yoo BC, Bubnov R, Hagan S, Danesi R, et al. EPMA position paper in cancer: current overview and future perspectives. EPMA J. 2015;6:9. https://doi.org/10.1186/s13167-0150030-6.

Publisher's note Springer Nature remains neutral with regard to jurisdictional claims in published maps and institutional affiliations. 